SLAC-PUB-16419

\title{
Compressing the Inert Doublet Model
}

\author{
Nikita Blinov $(a, b, c)$, Jonathan Kozaczuk ${ }^{(a)}$, \\ David E. Morrissey ${ }^{(a)}$, and Alejandro de la Puente ${ }^{(a, d)}$ \\ (a) TRIUMF, 4004 Wesbrook Mall, Vancouver, BC V6T 2A3, Canada \\ (b) Department of Physics and Astronomy, University of British Columbia, Vancouver, BC \\ V6T 1Z1, Canada \\ (c) SLAC National Accelerator Laboratory, 2575 Sand Hill Road, Menlo Park, CA, 94025, \\ $U S A$ \\ (d) Department of Physics, Carleton University, Ottawa, ON K1S 5B6, Canada \\ email: nblinov@slac.stanford.edu, jkozaczuk@triumf.ca, \\ dmorri@triumf.ca, apuente@physics.carleton.ca
}

July 24, 2018

\begin{abstract}
The Inert Doublet Model relies on a discrete symmetry to prevent couplings of the new scalars to Standard Model fermions. This stabilizes the lightest inert state, which can then contribute to the observed dark matter density. In the presence of additional approximate symmetries, the resulting spectrum of exotic scalars can be compressed. Here, we study the phenomenological and cosmological implications of this scenario. We derive new limits on the compressed Inert Doublet Model from LEP, and outline the prospects for exclusion and discovery of this model at dark matter experiments, the LHC, and future colliders.
\end{abstract}




\section{Introduction}

The Inert Doublet Model (IDM) extends the Standard Model (SM) with a scalar $S U(2)_{L}$ doublet that is odd under an unbroken $\mathbb{Z}_{2}$ symmetry [1]. This symmetry forbids direct couplings of the new doublet to the SM fermions while allowing couplings to the Higgs, and implies that the lightest state derived from the new doublet is stable. For these reasons, the IDM has been studied extensively as a model of dark matter (DM) 2, 3, as a simple module to investigate deviations in the properties of the Higgs boson [2, 4], and as a general source of new missing-energy signals in collider experiments [4, 5]. Many other scenarios that postulate additional weakly-charged scalars can also be mapped onto (one or many copies of) the IDM [6, 7, 8, 9, 10, 11, 12, 13, and the model can help to induce a strongly first-order electroweak phase transition suitable for electroweak baryogenesis [14, 15, 16, 17, 18, 19].

The scalar sector of the IDM contains two doublets $H_{1,2}$ transforming under $S U(3)_{c} \times$ $S U(2)_{L} \times U(1)_{Y}$ as $(\mathbf{1}, \mathbf{2}, 1 / 2)$ with a tree-level potential given by

$$
\begin{aligned}
V=\mu_{1}^{2}\left|H_{1}\right|^{2}+\mu_{2}^{2}\left|H_{2}\right|^{2}+\lambda_{1}\left|H_{1}\right|^{4}+\lambda_{2}\left|H_{2}\right|^{4} \\
\quad+\lambda_{3}\left|H_{1}\right|^{2}\left|H_{2}\right|^{2}+\lambda_{4}\left|H_{1}^{\dagger} H_{2}\right|^{2}+\frac{\lambda_{5}}{2}\left[\left(H_{1}^{\dagger} H_{2}\right)^{2}+\text { h.c. }\right] .
\end{aligned}
$$

We identify $H_{1}$ with the SM Higgs field and assume that only it gets a vacuum expectation value $(\mathrm{VEV})$ with $\left\langle H_{1}\right\rangle=v \simeq 174 \mathrm{GeV}$. The tree-level scalar mass spectrum is then

$$
\begin{aligned}
m_{h}^{2} & =-2 \mu_{1}^{2}=4 \lambda_{1} v^{2} \\
m_{H}^{2} & =\mu_{2}^{2}+\left(\lambda_{3}+\lambda_{4}+\lambda_{5}\right) v^{2} \\
m_{A}^{2} & =\mu_{2}^{2}+\left(\lambda_{3}+\lambda_{4}-\lambda_{5}\right) v^{2} \\
m_{H^{ \pm}}^{2} & =\mu_{2}^{2}+\lambda_{3} v^{2} .
\end{aligned}
$$

The SM-like Higgs boson $h$ comes entirely from $H_{1}$ while the rest come from $H_{2}$. The other components of $H_{1}$ are eaten by the weak vector bosons.

It is straightforward to check that Eq. (1) is the most general dimension-four scalar potential consistent with the discrete $\mathbb{Z}_{2}$ under which $H_{1} \rightarrow H_{1}$ and $H_{2} \rightarrow-H_{2}$ [20]. This symmetry also forbids $H_{2}$ from coupling directly to SM fermions. The form of the scalar potential is constrained further in the presence of additional approximate symmetries. Extending the $\mathbb{Z}_{2}$ to a global $U(1)$ acting only on $H_{2}$ forces $\lambda_{5}=0$ and leads to $m_{H}=m_{A}$ [2, while extending the $\mathbb{Z}_{2}$ to a global $S U(2)$ acting on $H_{2}$ forces both $\lambda_{4}=\lambda_{5}=0$ and makes all three exotic scalars degenerate [21]. These additional symmetries could be the parent symmetry of the stabilizing $\mathbb{Z}_{2}$, or they could arise from some other source. Motivated by these possible extended symmetries, we investigate the IDM in the limit of parametrically small $\left|\lambda_{4}\right|$ and $\left|\lambda_{5}\right|$. The resulting spectrum of exotic scalars is then compressed, which has significant phenomenological implications.

In this paper, we study the IDM in the compressed limit, focusing on the properties of IDM dark matter in this regime and the prospects for discovering the new scalars using existing and future collider data. Beginning in Section 2 we discuss the extent to which imposing additional approximate symmetries can produce a compressed mass spectrum in 
the IDM. Next, in Section 3 we review basic existing constraints on the IDM. In Section 4, we study the dark matter and cosmological limits on the theory with nearly mass-degenerate scalars. Sections 5, 6, and 7) investigate the current collider limits and future discovery prospects of the compressed IDM at LEP, the LHC, and proposed future colliders. Finally, Section 8 is reserved for our conclusions.

\section{Small Mass Splittings}

A compressed inert scalar spectrum arises for values of $\left|\lambda_{4}\right|$ and $\left|\lambda_{5}\right|$ much smaller than unity. From Eqs. (3 4 4 5 ) the mass splittings in this limit are

$$
\begin{aligned}
& \Delta^{0}=m_{A}-m_{H} \simeq-\frac{\lambda_{5} v^{2}}{m_{H}}, \\
& \Delta^{ \pm}=m_{H^{ \pm}}-m_{H} \simeq-\frac{\left(\lambda_{4}+\lambda_{5}\right) v^{2}}{2 m_{H}} .
\end{aligned}
$$

Small values of $\left|\lambda_{4}\right|$ and $\left|\lambda_{5}\right|$ can arise in a technically natural way if the theory has additional approximate symmetries. The three relevant symmetries in this regard are a global $U(1)$ acting on $H_{2}$, a global $S U(2)$ acting on $H_{2}$, and an extension of the custodial symmetry of the SM to include the inert doublet. We consider all three here, and investigate their implications for the mass spectrum of the new scalars and the lifetimes of the heavier states. Our primary conclusion is that the neutral mass splitting $\Delta^{0}$ can be arbitrarily small, while the charged mass splitting $\Delta^{ \pm}$tends to be larger than about a GeV.

\subsection{Symmetries and Splittings}

For $\lambda_{5}=0$ in the potential, Eq. (1), the theory has an enhanced symmetry under global $U(1)_{2}$ transformations acting on $H_{2}$ alone [2]. This leads to $m_{H}=m_{A}$, and it is convenient in this case to assemble these two real scalars into a single neutral complex scalar. Note as well that $U(1)_{2}$ is trivially non-anomalous and its generator commutes with all the gauge generators; thus it can be an exact symmetry of the theory.

With $\lambda_{4}=0=\lambda_{5}$, the scalar potential is also invariant under global $S U(2)_{2}$ transformations acting on $H_{2}$ alone. This leads to $m_{H}=m_{A}=m_{H^{ \pm}}$at tree-level. Both $\lambda_{4}$ and $\lambda_{5}$ can also be set to zero by extending the custodial symmetry of the SM to include the new doublet [21] 1] However, in contrast to the global $U(1)_{2}$ described above, both of these symmetries of the scalar potential are broken explicitly by gauging $S U(2)_{L} \times U(1)_{Y}$. Specifically, the $S U(2)_{2}$ generator does not commute with those of $S U(2)_{L}$, while $U(1)_{Y}$ breaks the extended custodial invariance just like in the SM.

Based on these considerations, it is technically natural for $\left|\lambda_{5}\right|$ to be arbitrarily small, while very suppressed values of $\left|\lambda_{4}\right|$ may require some degree of fine tuning. This can be

\footnotetext{
1 There is a one-parameter family of ways to extend the custodial symmetry. General choices of the embedding parameter force $\lambda_{4}=0=\lambda_{5}$, although special choices also allow $\lambda_{4}= \pm \lambda_{5}[21$.
} 


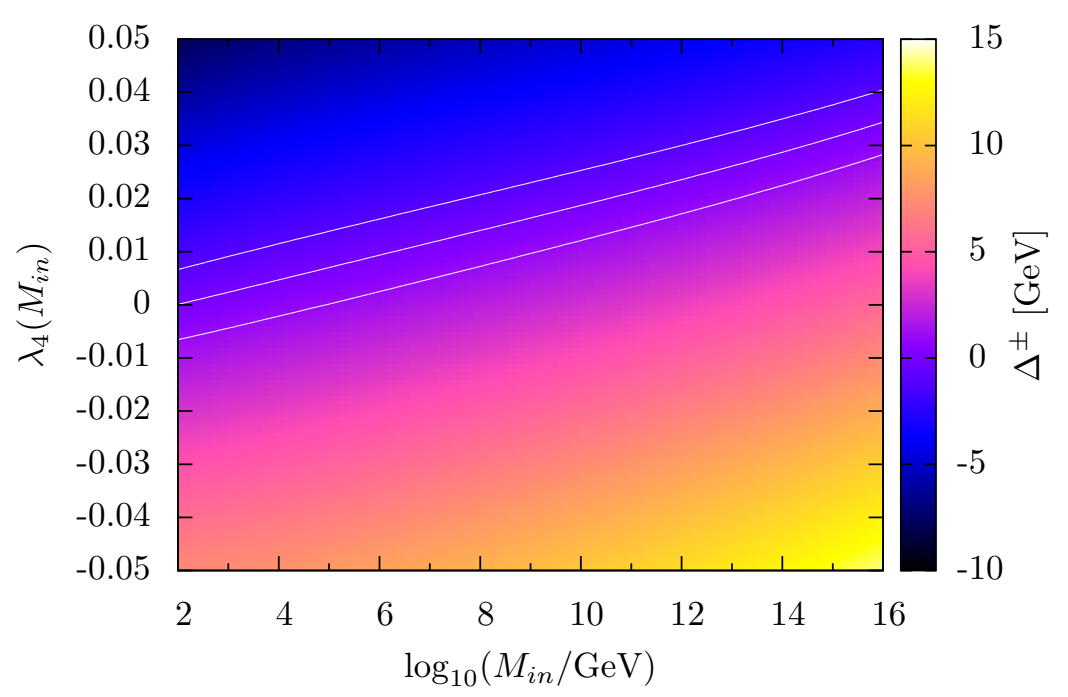

Figure 1: Tree-level charged-neutral mass splittings $\Delta^{ \pm}$for a given value of $\lambda_{4}\left(M_{\text {in }}\right)$ specified at the input scale $M_{\text {in }}$ with $m_{H}=100 \mathrm{GeV}, \lambda_{2}\left(m_{Z}\right)=0.1, \lambda_{3}\left(m_{Z}\right)=0.05$, and $\lambda_{5}=0$. The solid white lines show contours of $\Delta^{ \pm}=(0 \pm 1) \mathrm{GeV}$.

seen explicitly in the one-loop renormalization group $(R G)$ equations of these couplings [22]:

$$
\begin{aligned}
(4 \pi)^{2} \frac{d \lambda_{4}}{d t}= & -3 \lambda_{4}\left(3 g^{2}+g^{2}\right)+4 \lambda_{4}\left(\lambda_{1}+\lambda_{2}+2 \lambda_{3}+\lambda_{4}\right)+2 \lambda_{4}\left(3 y_{t}^{2}+3 y_{b}^{2}+y_{\tau}^{2}\right) \\
& +3 g^{2} g^{\prime 2}+8 \lambda_{5}^{2} \\
(4 \pi)^{2} \frac{d \lambda_{5}}{d t}= & -3 \lambda_{5}\left(3 g^{2}+g^{\prime 2}\right)+4 \lambda_{5}\left(\lambda_{1}+\lambda_{2}+2 \lambda_{3}+3 \lambda_{4}\right)+2 \lambda_{5}\left(3 y_{t}^{2}+3 y_{b}^{2}+y_{\tau}^{2}\right)
\end{aligned}
$$

where $t=\ln \left(\mu / \mu_{0}\right)$, and $\mu$ is the renormalization scale with $\mu_{0}=100 \mathrm{GeV}$. As expected, if $\lambda_{5}$ vanishes at any reference scale it will vanish at any other. In contrast, even if $\lambda_{4}=0=\lambda_{5}$ at some input scale $M_{i n}$, it will be regenerated by the $g^{2} g^{\prime 2}$ term in the second line of Eq. (8). The appearance of both $g$ and $g^{\prime}$ in this contribution coincides with the explicit breaking of both the $S U(2)_{2}$ and extended custodial symmetries by electroweak gauging.

To examine the tree-level mass splitting induced by the RG running of $\lambda_{4}$, we compute the low-scale mass difference $\Delta^{ \pm}$between $H^{ \pm}$and $H^{0}$ assuming small initial values of $\lambda_{4}\left(M_{i n}\right)$ defined at the high input scale $M_{i n}$. The RG evolution is implemented using the equations for all the relevant couplings tabulated in Ref. [22]. In doing so, we fix the gauge and Yukawa couplings and $\lambda_{1}$ according to their measured values, and we set $m_{H}=100 \mathrm{GeV}$, $\lambda_{2}\left(m_{Z}\right)=0.1, \lambda_{3}\left(m_{Z}\right)=0.05$, and $\lambda_{5}=0$. The results are mostly independent of these choices provided they are much smaller than the electroweak gauge couplings. The resulting RG-induced low-scale mass splitting $\Delta^{ \pm}$is shown in Fig. 1, with the solid lines indicating where $\Delta^{ \pm}=(0 \pm 1) \mathrm{GeV}$. We find typical mass splittings on the order of a few $\mathrm{GeV}$ for vanishing initial values of $\lambda_{4}\left(M_{i n}\right)$ and $\lambda_{5}\left(M_{i n}\right)$ unless $M_{i n}$ is very close to the weak scale.

In addition to the RG-induced mass splitting, there is also a finite contribution to $\Delta^{ \pm}$ 
from electroweak symmetry breaking. At one-loop order it is [23]

$$
\Delta_{\text {one-loop }}^{ \pm}=\frac{\alpha_{W} s_{W}^{2}}{4 \pi} m_{H^{ \pm}} f\left(m_{Z} / m_{H^{ \pm}}\right)
$$

where the function $f(x)$ is given by

$$
f(x)=-\frac{x}{4}\left(2 x^{3} \ln x+\left(x^{2}-4\right)^{3 / 2} \ln \left[\left(x^{2}-2-x \sqrt{x^{2}-4}\right) / 2\right]\right) .
$$

Note that we have already accounted for the UV-divergent part listed in Ref. [23] by the RG analysis above. We find that this additional splitting is less than about $\Delta^{ \pm} \lesssim 100 \mathrm{MeV}$ for the parameter regions of greatest interest from the standpoint of collider searches, $m_{H} \lesssim$ $100 \mathrm{GeV}$, and is almost always subdominant to the RG-induced tree-level splitting considered above.

Based on these results, in the remainder of this paper we will study the IDM in the compressed limit with arbitrarily small mass splittings $\Delta^{0}$ between the neutral $H$ and $A$ states, but we will assume somewhat larger mass splittings among these and the charged state, with $\left|\Delta^{ \pm}\right| \gtrsim 1 \mathrm{GeV}$. Reducing $\Delta^{ \pm}$much below this typically requires either a fortuitous accident or a significant fine-tuning for $M_{i n} \gg m_{Z}$. We will also assume $\Delta^{0} \geq 0$ with no loss of generality, as well as $\Delta^{ \pm}>0$ to avoid a stable charged relic.

\subsection{Decay Lifetimes}

A compressed mass spectrum in the IDM can suppress the decay rates of the heavier states down to the lightest. As mentioned above, we will consider arbitrarily small neutral splittings $\Delta^{0}$ but we will focus on charged mass splittings above $\Delta^{ \pm} \gtrsim 1 \mathrm{GeV}$. These values generally cause the $H^{ \pm}$decay promptly on collider time scales but allow the heavier neutral state $A$ to be long-lived on both collider and cosmological time scales.

The dominant decay channels for both $A$ and $H^{ \pm}$are $A \rightarrow H Z^{*}$ and $H^{ \pm} \rightarrow H W^{ \pm *}$, with off-shell weak vector bosons. When $\Delta^{0, \pm} \ll m_{W}$, the vector bosons can be integrated out to give the leading effective interactions

$$
\begin{aligned}
-\mathscr{L}_{\text {eff }} \supset \frac{g^{2}}{2 m_{W}^{2}} & {\left[A \stackrel{\leftrightarrow}{\partial_{\mu}} H \sum_{i} \bar{f}_{i} \gamma^{\mu}\left(a_{V}^{i}+a_{A}^{i} \gamma^{5}\right) f_{i}\right.} \\
+ & \left.i H^{+} \stackrel{\leftrightarrow}{\partial_{\mu}}(H-i A) \sum_{j k} \bar{f}_{j} \gamma^{\mu}\left(c_{V}^{j k}+c_{A}^{j k} \gamma^{5}\right) f_{k}^{\prime}+\text { (h.c.) }\right],
\end{aligned}
$$

where the couplings are given by

$$
a_{v}^{i}=\frac{1}{2}\left(t_{i}^{3}-2 Q_{i} s_{W}^{2}\right), \quad a_{A}^{i}=-\frac{1}{2} t_{i}^{3},
$$

with $i$ running over all SM fermion species, and

$$
\begin{aligned}
c_{V}^{j k}=-c_{A}^{j k} & =\frac{1}{2 \sqrt{2}} \delta^{j k} & & \text { (leptons) } \\
& =\frac{1}{2 \sqrt{2}} V_{\mathrm{CKM}}^{j k} & & \text { (quarks) }
\end{aligned}
$$


where $j$ runs over up-type fermions and $k$ over down-type, and $V_{\mathrm{CKM}}$ is the CabbiboKobayashi-Maskawa matrix.

The decay width for $A \rightarrow H+f \bar{f}$ derived from these interactions is approximately

$$
\Gamma(A \rightarrow H)=\frac{1}{120 \pi^{3}} \frac{g^{4}}{m_{W}^{4}}\left(\Delta^{0}\right)^{5} \sum_{i} N_{c}^{i}\left[\left(a_{V}^{i}\right)^{2}+\left(a_{A}^{i}\right)^{2}\right] \times \Theta\left(\Delta^{0}-2 m_{i}\right)
$$

with $N_{c}^{i}$ the number of colours of the $i$-th species. Similarly, the width for the charged $H^{+} \rightarrow H+f \bar{f}^{\prime}$ channels is

$$
\Gamma\left(H^{+} \rightarrow H\right)=\frac{1}{120 \pi^{3}} \frac{g^{4}}{m_{W}^{4}}\left(\Delta^{ \pm}\right)^{5} \sum_{j k} N_{c}^{j}\left[\left|c_{V}^{j k}\right|^{2}+\left|c_{A}^{j k}\right|^{2}\right] \times \Theta\left(\Delta^{ \pm}-m_{j}-m_{k}\right)
$$

A similar expression applies for $H^{+} \rightarrow A f \bar{f}^{\prime}$ decays.

These expressions are only sensible when the hadronic final states can be reliably treated as partons. In our numerical estimates, we apply them down to $\Delta=2 \mathrm{GeV}$. For smaller splittings, the hadronic decays can be handled similarly to tau leptons [24] or nearlydegenerate electroweakino superpartners [25, 26, 27]. No hadronic modes are available for $\Delta<m_{\pi}$, while the one-pion mode $A \rightarrow H \pi^{0}\left(H^{+} \rightarrow H \pi^{+}\right)$is expected to dominate the hadronic width for $m_{\pi}<\Delta \lesssim 1 \mathrm{GeV}$ [27]. Thus, to estimate the hadronic decay width for $m_{\pi}<\Delta<2 \mathrm{GeV}$ we compute the one-pion width explicitly up to $1 \mathrm{GeV}$ and then interpolate the result smoothly to the partonic result at $2 \mathrm{GeV}$. A more detailed treatment following Refs. [24, 27] could be applied if specific partial widths are desired.

To compute the one-pion decay widths, we match the quark-level operators of Eq. (12) to the axial isospin currents in chiral perturbation theory and apply [28]

$$
\left\langle 0\left|j_{5}^{\mu a}\right| \pi^{b}(p)\right\rangle=i f_{\pi} p^{\mu} \delta^{a b}
$$

with $f_{\pi}=93 \mathrm{MeV}$. This gives the one-pion width

$$
\Gamma\left(A \rightarrow H \pi^{0}\right)=\frac{1}{128 \pi} \frac{g^{4}}{m_{W}^{2}} f_{\pi}^{2} \frac{p}{m_{A}^{2}}\left(m_{A} E_{\pi}+E_{H} E_{\pi}+p^{2}\right) \simeq \frac{1}{32 \pi} \frac{g^{2}}{m_{W}^{2}} f_{\pi}^{2} \Delta^{3},
$$

where $E_{i}=\sqrt{m_{i}^{2}+p^{2}}$ and

$$
p^{2}=\frac{1}{4 m_{A}^{2}}\left(m_{A}^{4}+m_{H}^{4}+m_{\pi}^{4}-2 m_{A}^{2} m_{H}^{2}-2 m_{A}^{2} m_{\pi}^{2}-2 m_{H}^{2} m_{\pi}^{2}\right) .
$$

The same expression applies to $\Gamma\left(H^{+} \rightarrow H \pi^{+}\right)$after replacing $\Delta^{0} \rightarrow \Delta^{ \pm}$and $m_{A} \rightarrow m_{H^{ \pm}}$ and adding a factor of $\left|V_{\mathrm{CKM}}^{u d}\right|^{2}$.

The total lifetimes of $A$ and $H^{+}$as a function of the mass splittings are shown in Fig. 2 . The mass of $H$ is set to $m_{H}=70 \mathrm{GeV}$ in generating this figure, but the lifetimes are not sensitive to this value provided $m_{H} \gg \Delta$. For $H^{+}$decays, we assume $\Delta^{ \pm} \gg \Delta^{0}$ and include decays to both $H$ and $A$ final states. We also consider only $\Delta^{ \pm}>m_{e}$ since otherwise $H^{+}$ 


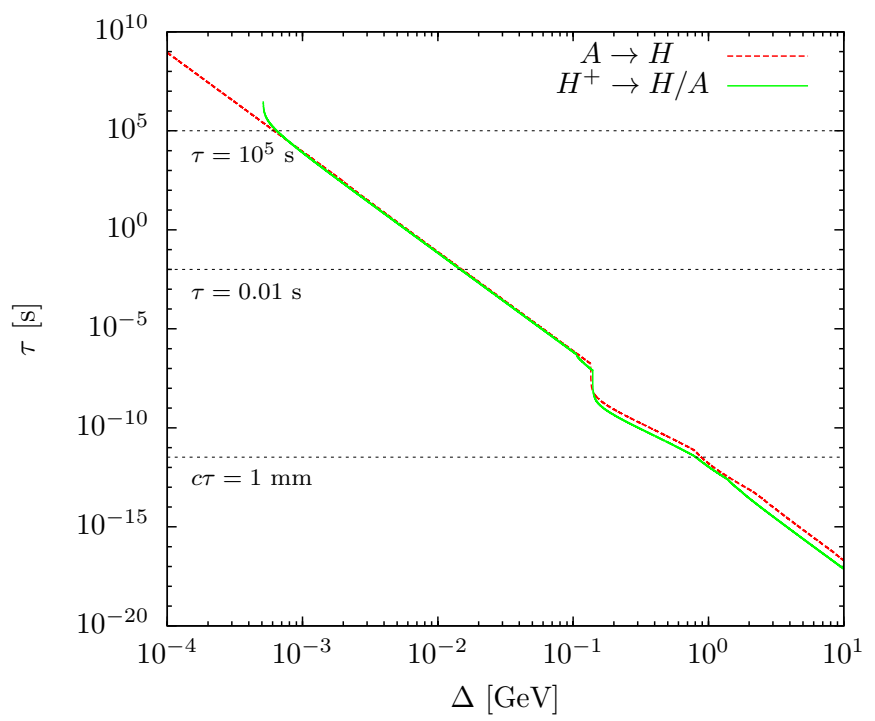

Figure 2: Decay lifetimes of the neutral $A$ and charged $H^{ \pm}$states as a function of the mass splitting $\Delta$ with the lightest $H$ state.

is stable (and in any case, the results of the previous section suggest that splittings this small are highly unlikely). For neutral decays, we take $\Delta^{ \pm}>\Delta^{0}$ and allow for much smaller neutral splittings. The neutral decay width below $\Delta^{0}<2 m_{e}$ is due to neutrino final states. ${ }^{2}$ For both the neutral and charged states, we see from Fig. 2 that the decays are prompt on collider time scales, $c \tau<1 \mathrm{~mm}$, for mass splittings above a few hundred $\mathrm{MeV}$. Some potential implications of a long-lived neutral state for cosmology will be discussed below.

\section{Basic Bounds}

The parameters of the IDM are constrained by a number of direct and indirect bounds. By assumption, the model should produce a locally stable electroweak vacuum with only $H_{1}$ obtaining a VEV. This implies [2, 4]

$$
\lambda_{1,2}>0, \quad \lambda_{3},\left(\lambda_{3}+\lambda_{4}-\left|\lambda_{5}\right|\right)>-2 \sqrt{\lambda_{1} \lambda_{2}} .
$$

The standard electroweak vacuum will also be globally stable if

$$
\mu_{1}^{2} / \sqrt{\lambda_{1}}<\mu_{2}^{2} / \sqrt{\lambda_{2}} \text {. }
$$

Note that $\mu_{1}^{2}<0$ is typically needed for EWSB. It is also standard to demand that the scalar couplings be small enough to maintain perturbativity to at least a few times the weak scale. For masses of the charged and neutral scalars that are relatively close to one another,

\footnotetext{
${ }^{2}$ Note as well that the radiative decay $A \rightarrow H+\gamma$ is forbidden by angular momentum conservation given the point interaction of Eq. (12), in contrast to the decay $\chi_{2}^{0} \rightarrow \chi_{1}^{0} \gamma$ that can occur for neutralinos in supersymmetric theories [29, 30].
} 
corresponding to small $\lambda_{4}$ and $\lambda_{5}$, these conditions are met easily for moderate values of $\lambda_{3}$ and positive values of $\mu_{2}^{2}$.

New electroweakly-charged states can modify precision electroweak observables. The leading indirect effect can be described as a shift in $\Delta T$ relative to the SM (including a SM Higgs of mass $m_{h}=125 \mathrm{GeV}$ ),

$$
\Delta T=\frac{1}{32 \pi^{2} \alpha v^{2}}\left[F\left(m_{H^{ \pm}}^{2}, m_{A}^{2}\right)+F\left(m_{H^{ \pm}}^{2}, m_{H}^{2}\right)-F\left(m_{A}^{2}, m_{H}^{2}\right)\right],
$$

where $F(x, y)=(x+y) / 2-x y \ln (x / y) /(x-y)$. This shift in $\Delta T$ can usually be approximated by 31

$$
\Delta T \simeq \frac{1}{24 \pi^{2} \alpha v^{2}}\left(m_{H^{ \pm}}-m_{A}\right)\left(m_{H^{ \pm}}-m_{H}\right) .
$$

This is clearly suppressed in the compressed limit we are considering, and does not produce a relevant constraint given the currently allowed range $T=0.01 \pm 0.12$ [32]

A more direct electroweak requirement is that the SM $W^{ \pm}$and $Z^{0}$ vector bosons should not be able to decay significantly to the exotic scalars. Barring extreme kinematic suppression, this implies

$$
m_{H}+m_{A}>m_{Z}, \quad m_{A}+m_{H^{ \pm}}, m_{H}+m_{H^{ \pm}}>m_{W}
$$

The limit for $Z$ decays comes from direct searches for $Z \rightarrow f \bar{f}+\nu \bar{\nu}$, and bounds the process $Z \rightarrow H A$ with $A \rightarrow Z^{*} H$ with $Z^{*} \rightarrow f \bar{f}$ [4. A similar but slightly weaker bound can be obtained from the invisible decay width of the $Z$, and is applicable when both $A$ and $H$ are long-lived, stable, or have very soft decay products [4]. Limits from $W$ decays to $A H^{ \pm}$or $H H^{ \pm}$can presumably be obtained as well, but will be weaker than the $Z$-decay bound for $\Delta^{ \pm}>\Delta^{0}$.

Decays of the SM-like Higgs to light exotic scalars can easily overwhelm the narrow Higgs width to SM modes. The partial Higgs decay rate to a light scalar is [4]

$$
\Gamma(h \rightarrow S S)=\xi_{S}\left(\frac{m_{S}^{2}-\mu_{2}^{2}}{m_{h}}\right) \sqrt{1-4 m_{S}^{2} / m_{h}^{2}},
$$

where $S=H, A, H^{ \pm}$and $\xi_{S}=1(2)$ for $S=H, A\left(H^{ \pm}\right)$. A recent analysis of LHC Higgs (rate) data applied to the IDM finds

$$
\left|m_{H}^{2}-\mu_{2}^{2}\right| / 2 v^{2}=\left|\lambda_{3}+\lambda_{4}+\lambda_{5}\right| / 2 \lesssim 0.012(0.007),
$$

assuming that only $h \rightarrow H H$ is open and that $m_{H}=60 \mathrm{GeV}(10 \mathrm{GeV})$ [33]. When more than one channel is accessible, we expect a corresponding bound to apply to the orthogonal sum of the effective couplings. Note that the RG evolution of $\lambda_{3}$ is inhomogeneous and sourced by gauge interactions (much like $\lambda_{4}$ ), and one would typically expect values of $\lambda_{3}$ that are too large to satisfy the condition of Eq. (27).

Even with $m_{H}>m_{h} / 2$, the IDM can alter the decays of the SM Higgs boson through loop effects. In particular, the charged scalar modifies the width for $h \rightarrow \gamma \gamma$ at one-loop with the contribution to the amplitude depending on $\lambda_{3}$ and $m_{H^{ \pm}}$[4]. This only provides a moderate bound on $\left|\lambda_{3}\right| \lesssim 1$ for $m_{H^{+}}>m_{Z} / 2$ [33, 34, 35]. 


\section{Cosmological Constraints}

The IDM contains a promising dark matter (DM) candidate provided the lightest new scalar state is neutral. A compressed mass spectrum in the IDM has important implications for the relic density and detection prospects of this DM component. Very small mass splittings can also lead to long-lived metastable states whose late decays may be cosmologically important. We examine these topics in this section.

\subsection{Relic Abundances}

Thermal dark matter production in the IDM has been studied extensively [2, 3, 4, 5, 20, 22, 36, 37, 38, 39, 40, 41]. We extend these works by elucidating the key dynamics in the compressed regime. An important implication of the compressed spectrum is that coannihilation plays a central role in determining the relic density of the lightest state, which we assume to be $H$. Again, for our analysis we assume a small mass splitting between the neutral DM states with $\Delta^{0} \sim 100 \mathrm{keV}$ (motivated by direct detection constraints in Sec. 4.2), but we consider larger mass splittings between the charged and neutral states, $\Delta^{ \pm} \geq 1 \mathrm{GeV}$. As shown in Sec. 2, the coupling $\lambda_{5}$ and therefore $\Delta^{0}$ can be taken to be arbitrarily small in a technically natural way. The specific value of $\Delta^{0}$ does not matter much for the relic density provided it is much smaller than the freeze-out temperatures we obtain ${ }^{3}$, since in this case coannihilation between the $H^{0}$ and $A^{0}$ states will not receive any significant Boltzmann suppression. In contrast, the charged-neutral splitting $\Delta^{ \pm}$is sourced by gauge couplings in the course of RG running and can be significantly larger. Thus, we examine the effects of larger $\Delta^{ \pm}$on the DM relic density.

We compute the relic abundance of $H$ as a function of its mass for various choices of the mass splittings $\Delta^{0}$ and $\Delta^{ \pm}$and the Higgs coupling parameter $\lambda_{L}=\left(\lambda_{3}+\lambda_{4}+\right.$ $\left.\lambda_{5}\right) / 2$. The calculation is performed using micrOMEGAs 4.1.8[43], which includes the effects of coannihilation as well as two-, three-, and four-body annihilation channels. Our main results are summarized in Fig. 3. The left panel of this figure shows the impact of the chargedneutral splitting $\Delta^{ \pm}$on the abundance for fixed $\lambda_{L}$, while the right panel shows the effect of varying $\lambda_{L}=0.01,0.1,1$ with fixed $\Delta^{ \pm}=10 \mathrm{GeV}$. Note that for $m_{H} \leq m_{h} / 2$, these sizable $\lambda_{L}$ will typically induce an unacceptable $h \rightarrow$ invisible decay rate as discussed in Sec. 3 , Eq. (27). Also shown in both panels, by the horizontal dashed line, is the $1 \sigma$ upper limit on the dark matter density $\Omega_{\mathrm{cdm}} h^{2} \lesssim 0.1227$ as determined by the Planck collaboration [42].

The relic abundances in Fig. 3 can be understood in terms of coannihilation, resonances, and Higgs couplings. Coannihilation usually relies on gauge couplings, so when it is important the abundance curves for different values of $\lambda_{L}$ collapse. This can be seen near the $Z$ resonance at $m_{H}=m_{Z} / 2$ where $A-H$ coannihilation dominates. A Higgs funnel at $m_{H}=m_{h} / 2$ that depends on $\lambda_{L}$ is also visible in Fig. 3. The relic abundance below $m_{H} \lesssim 40 \mathrm{GeV}$ is very sensitive to the mass splitting $\Delta^{ \pm}$, as can be seen in the left panel of Fig. 3. Here, coannihilation with $H^{ \pm}$plays a leading role for small mass differences because

\footnotetext{
${ }^{3}$ For $m_{H}>1 \mathrm{GeV}$ typical freeze-out temperatures are $T_{\mathrm{fo}} \sim m_{H} / 20 \gg \Delta^{0}$.
} 

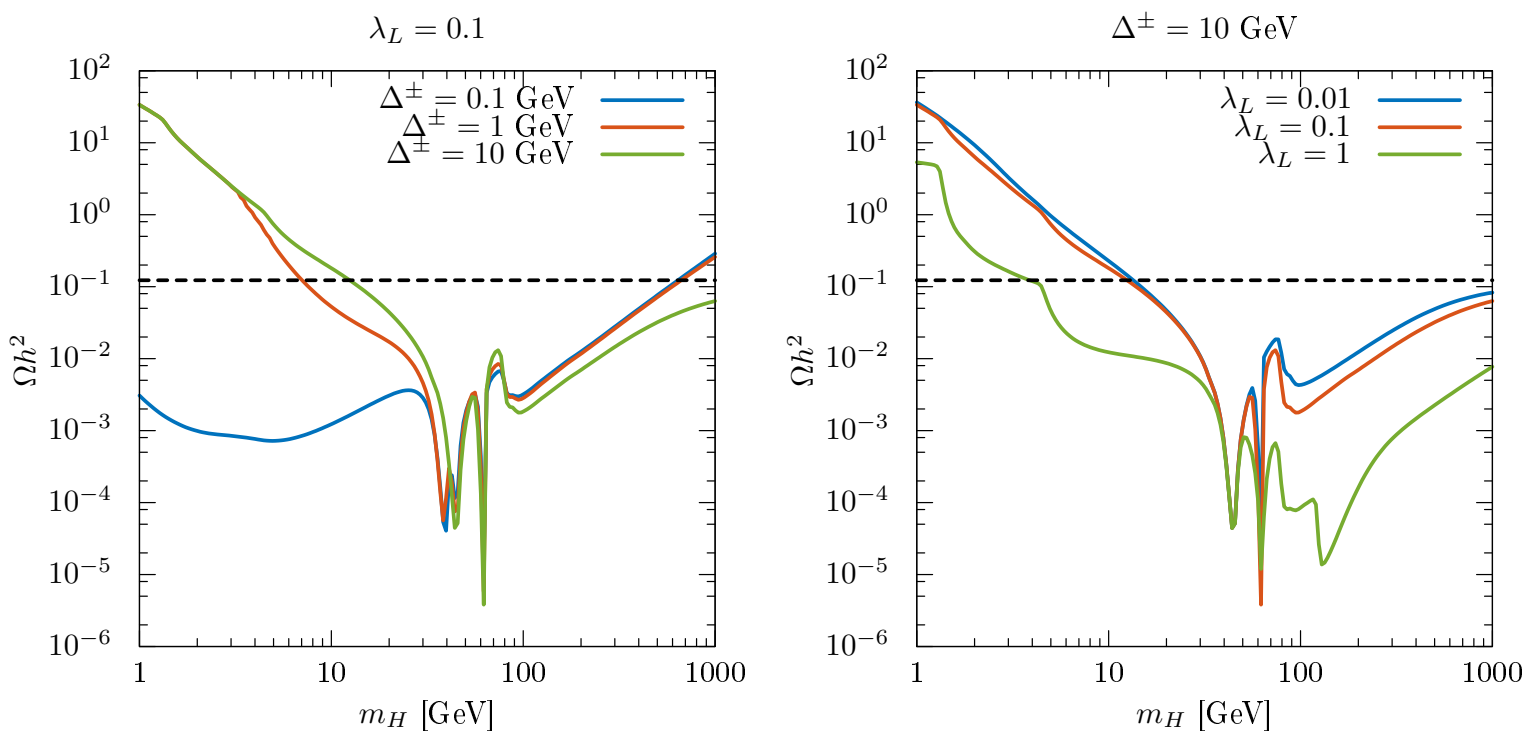

Figure 3: Abundance of IDM dark matter as a function of mass in the compressed regime. The left panel shows the abundance for fixed $\lambda_{L}=0.1$ and charged-neutral mass splittings of $\Delta^{ \pm}=0.1,1.0,10 \mathrm{GeV}$. The right panel shows the relic abundance for $\lambda_{L}=0.01,0.1,1.0$ and fixed splitting $\Delta^{ \pm}=10 \mathrm{GeV}$. The horizontal dashed line in both panels is the $1 \sigma$ upper limit on the total dark matter density as determined by the Planck collaboration [42].

the charged state has the direct two-body annihilation channel $H^{+} H^{-} \rightarrow \gamma \gamma$ open, while the neutral states must go through three- and four-body channels in this region.

At higher masses, the dominant annihilation process is into pairs of weak vector bosons [20]. Annihilation into off-shell vector bosons can also dominate below threshold [37], (and is included in the version of micrOMEGAs 4.1.8 [43] we use). The relative contributions of transverse and longitudinal final states depend on the scalar couplings $\lambda_{i>2}$, with annihilation to longitudinal modes enhanced for larger values of these couplings [20] as expected from the Goldstone equivalence theorem [44, 45]. In the compressed regime, $\left|\lambda_{4}\right|$ and $\left|\lambda_{5}\right|$ are both assumed to be small, leaving $\lambda_{3} \simeq 2 \lambda_{L}$ as the only potentially large one. The enhancement of the annihilation rate with larger $\lambda_{L}$ (and to a lesser extent with larger $\Delta^{ \pm}$corresponding to increased $\left.\left|\lambda_{4}\right|\right)$ is clear in Fig. 3. When all the scalar couplings are small, the observed relic density is obtained from $m_{H} \simeq 535 \mathrm{GeV}[20]$.

\subsection{Dark Matter Detection}

The analysis above shows that for $m_{Z} / 2 \lesssim m_{H} \lesssim 500 \mathrm{GeV}$, the relic $H$ abundance can only make up a fraction of the total DM density when the scalar spectrum is compressed. Despite this, direct detection limits place strong additional constraints on the IDM relic component. Indirect detection does not appear to provide any further constraint.

Direct detection rules out the IDM for neutral mass splittings with $\Delta^{0} \lesssim 100 \mathrm{keV}$ for 

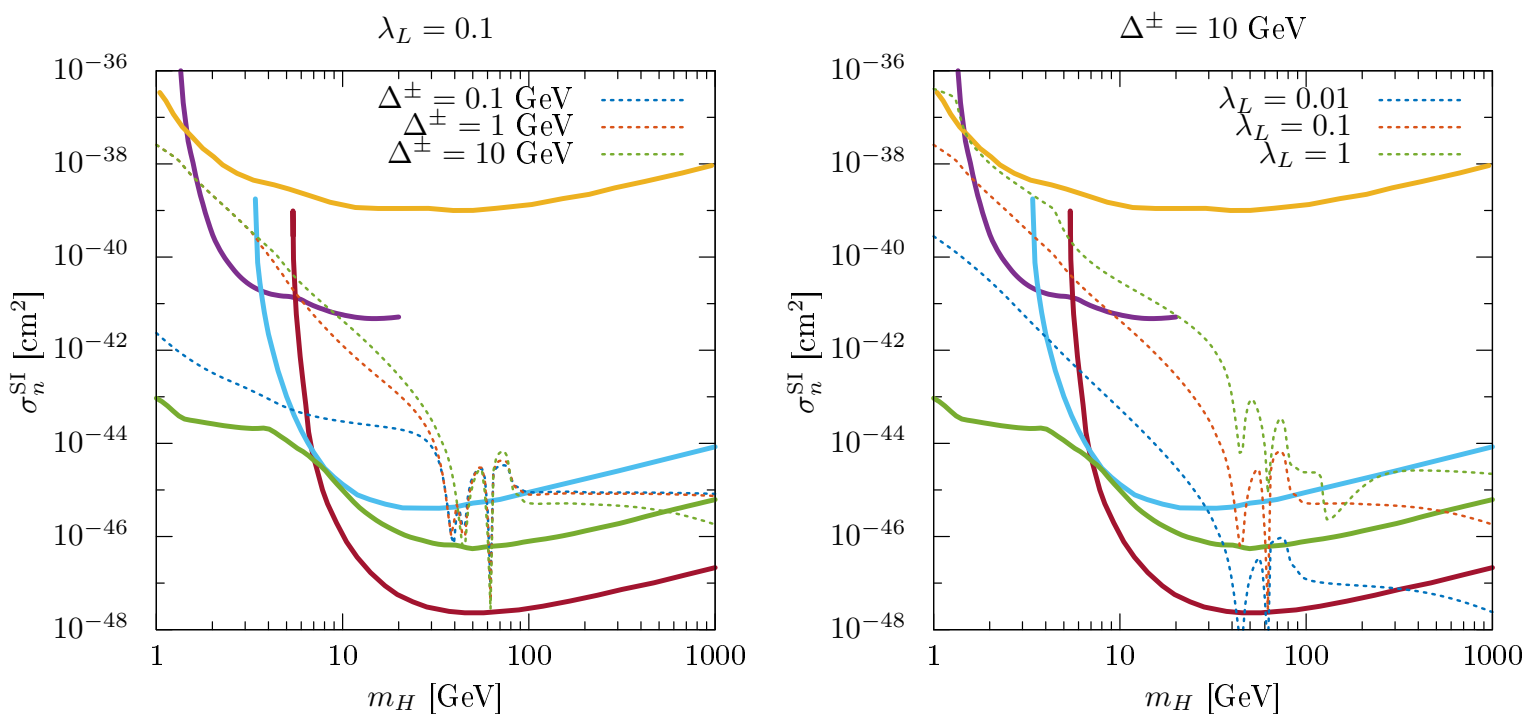

Figure 4: Limits on the effective DM-nucleon cross section as a function of mass from direct detection experiments and predictions for the IDM relic scaled by the expected thermal relic density. The solid lines correspond to the bounds from LUX [51] (light blue), CDMSLite [52] (purple), CRESST-Si [53] (yellow) and projected sensitivities of LZ (red) [54] and SuperCDMS SNOLAB (green) [55]. The dashed lines show the nucleon-scattering cross sections of $H$ rescaled by $\Omega / \Omega_{\mathrm{cdm}}$, with the colour coding identical to Fig. 3 .

$m_{H}>m_{Z} / 2$ [2]. In this highly degenerate limit, there is unsuppressed inelastic spinindependent scattering with nuclei, $H N \rightarrow A N$ [46, 47], via a vector interaction through the $Z$ with a very high cross section. This process turns off for neutral splittings above $\Delta^{0} \gtrsim 200 \mathrm{keV}$, and we will assume values at least this large for the remainder of the analysis.

In the presence of such a splitting between $H$ and $A$, the dominant interaction between IDM relics in the halo and nuclei is spin-independent (SI) elastic scattering through SM Higgs exchange. The resulting DM-nucleon cross-section is [48, 49]

$$
\sigma_{n}=\frac{\lambda_{L}^{2}}{\pi}\left(\frac{m_{n}}{m_{H}+m_{n}}\right)^{2} \frac{f_{n}^{2}}{m_{h}^{4}},
$$

where

$$
f_{n}=\sum_{q=u, d, s} m_{q}\langle n|\bar{q} q| n\rangle-\frac{\alpha_{s}}{4 \pi}\left\langle n\left|G_{\mu \nu}^{a} G^{a \mu \nu}\right| n\right\rangle .
$$

These matrix elements can evaluated using the results of Ref. [50].

The resulting bounds on the IDM relic $H$ from spin-independent nucleon scattering are shown in Fig. 4 as functions of the mass $m_{H}$. In both panels, the solid lines indicate the current limits from direct detection experiments, with the best current bounds for $m_{H} \gtrsim$ $5 \mathrm{GeV}$ from the LUX experiment [51] (light blue), along with CDMSLite [52] (purple) and CRESST-Si [53] (yellow) dominating at lower masses. We also show the projected 
sensitivities of LZ (red) [54] and SuperCDMS SNOLAB (green) [55]. The dashed lines in Fig. 4 show the nucleon-scattering cross sections of $H$ rescaled by $\Omega / \Omega_{\text {cdm }}$, with the colour coding identical to Fig. 3. Comparing these contours, we see that a light IDM relic component with $m_{Z} / 2<m_{H}<100 \mathrm{GeV}$ can be consistent with current bounds from direct detection. However, these bounds also suggest that $\left|\lambda_{L}\right|$ should be much smaller than unity in this mass region unless the relic density of $H$ is particularly small. Constraints from direct detection are easier to satisfy for larger $H$ masses, including $m_{H} \gtrsim 500 \mathrm{GeV}$ where the IDM relic can potentially make up the full DM relic density.

In contrast to direct detection, indirect detection signals of the IDM relic for $m_{H} \lesssim$ $500 \mathrm{GeV}$ are typically well below current sensitivities [40, 56, 59]. This is mainly due to the sub-critical density of $H$, but also from the much greater effectiveness of coannihilation during freeze out compared to today. Capture and annihilation of the $H$ state in the Sun or Earth is more promising [57, 58, but limits from direct detection are typically much stronger [58].

\subsection{Late-Time Decays}

The heavier IDM scalars can become long-lived on cosmological timescales for small mass splittings. From Fig. 2, we see that the decay lifetimes exceed $\tau \gtrsim 0.01 \mathrm{~s}$ for splittings below $\Delta \lesssim 20 \mathrm{MeV}$. Such decays can disrupt primordial nucleosynthesis (BBN) and inject additional energy into the cosmic microwave background (CMB).

Mass splittings below $\Delta \lesssim 20 \mathrm{MeV}$ are only expected for the neutral states, so we will focus on $A \rightarrow H Z^{*}$ modes. The dominant final states in this case are $e^{+} e^{-}$and $\nu \bar{\nu}$, with no hadronic component present. Comparing to the direct bounds on late-time electromagnetic energy injection from Ref. [60, 61], which only become significant for $\tau>10^{5} \mathrm{~s}$, we do not find a limit from BBN. Additionally, the energy released per decay is usually too small to destroy light nuclei. Very late decays with $\Delta^{0}<1 \mathrm{MeV}$ will produce mostly neutrinos, but can also yield photons through loops. These photons can disrupt the black body spectrum of the CMB [62]. However, given the expected primordial yield of $A$ and photonic branching fraction, the analysis of Ref. [63] indicates that no CMB limit is obtained for $\Delta^{0}>100 \mathrm{keV}$, as required by direct detection.

\section{$5 \quad$ LEP Limits}

Data from LEP can probe the IDM beyond the basic bounds outlined in Section 3 . The dominant production modes for the new scalars at LEP are $e^{+} e^{-} \rightarrow H^{+} H^{-}$via a $\gamma$ or $Z$, and $e^{+} e^{-} \rightarrow H A$ via a $Z$. Once created, the heavier states will decay down to the lightest neutral state (assumed to be $H$ here) through $H^{ \pm} \rightarrow H W^{ \pm *}$ and $A \rightarrow H Z^{*}$, with $H$ escaping the detector as missing energy. These features are very similar to the signals of supersymmetry in $\chi_{1}^{+} \chi_{1}^{-}$and $\chi_{2}^{0} \chi_{1}^{0}$ production, and thus LEP searches for electroweak superpartners are natural to apply to the IDM. 
A detailed analysis of this kind was undertaken in Ref. [31], where LEP searches for $\chi_{1}^{0} \chi_{2}^{0}$ in the DELPHI experiment [64] were used to derive an exclusion on $H A$ production 4 The limits found in this work extend up to $m_{A} \simeq 100 \mathrm{GeV}$ for larger neutral mass splittings, but there is no improvement over the $Z$ decay bound $\left(m_{A}+m_{H}>m_{Z}\right)$ for $\Delta^{0}<8 \mathrm{GeV}$. For the charged states, a reinterpretation of the OPAL results of Refs. [65, 66] was made in Ref. [67] to derive a limit of $m_{H^{ \pm}} \gtrsim 70-90 \mathrm{GeV}$. However, this analysis was approximate, and is only applicable to mass differences $\Delta^{ \pm} \gtrsim 5 \mathrm{GeV}$.

In this section we refine the estimate of the limits from LEP on $\mathrm{H}^{+} \mathrm{H}^{-}$production by reinterpreting searches for $\chi_{1}^{+} \chi_{1}^{-}$production. We also apply LEP searches using monophotons to test highly-compressed IDM spectra. Throughout our analysis, we assume $\Delta^{ \pm} \gg \Delta^{0}$ and $\Delta^{0} \lesssim 0.5 \mathrm{GeV}$. This implies that the $H$ and $A$ states are not constrained by the LEP reanalysis of Ref. [31], that the products of $A \rightarrow H Z^{*}$ decays are largely invisible to the LEP detectors, and that the $H^{+} \rightarrow A W^{*}$ and $H^{+} \rightarrow H W^{*}$ modes are effectively indistinguishable.

\subsection{General Searches for $H^{+} H^{-}$}

Experiments at LEP looked for supersymmetric chargino $\chi_{1}^{+} \chi_{1}^{-}$production in final states with leptons or jets and missing energy. The OPAL search of Ref. [65], using $680 \mathrm{pb}^{-1}$ of data at center-of-mass energies of 183-209 GeV, focused on dileptons and missing energy, with each chargino assumed to decay through an off-shell $W$ via $\chi_{1}^{+} \rightarrow \chi_{1}^{0} W^{*}$. Exactly the same decay topology occurs for $H^{+} H^{-}$production in the IDM, with $H^{+} \rightarrow H W^{*}$ and $H^{+} \rightarrow A W^{*}$, and thus the OPAL result can be used to constrain the IDM as well. Similar chargino searches were performed by the other LEP collaborations [66, 68], and were combined in Ref. [69], but only Ref. [65] stated their results with enough resolution to be useful for the present analysis.

The OPAL search of Ref. 65] concentrated on final states with dileptons and missing energy. Their exclusions are given in terms of limits on the inclusive $\chi_{1}^{+} \chi_{1}^{-}$production cross section at $\sqrt{s}=208 \mathrm{GeV}$ times the square of the branching fraction $B R\left(\chi_{1}^{+} \rightarrow \chi_{1}^{0} \nu_{\ell} \ell^{+}\right)$, with data taken at different collision energies re-weighted with a factor of $\beta / s$ (where $\beta=\sqrt{1-4 m_{H^{ \pm}}^{2} / s}$ ). To translate these limits to the IDM, we calculate the total $H^{+} H^{-}$ production cross section at $\sqrt{s}=208 \mathrm{GeV}$ and the leptonic branching fraction $B R\left(H^{+} \rightarrow\right.$ $H \nu_{\ell} \ell^{+}$) in Madgraph5 [70]. For $\Delta^{ \pm} \gg \Delta^{0}$, which we assume here, the same branching ratio is expected for $H^{+} \rightarrow A W^{*}$. To account for the different threshold behaviour of scalar production $\left(\beta^{3} / s\right)$ relative to fermion production $(\beta / s)$, we apply an additional re-weighting factor to account for the multiple collision energies used in the OPAL analysis [65]. We then apply the limits quoted in Ref. [65] to the corresponding combination in the IDM to derive exclusions on the IDM parameter space.

Figure 5 shows the $95 \%$ CL exclusion derived from our reanalysis in the $m_{H^{ \pm}}-m_{H}$ plane by the solid black contour, with the colour map showing the ratio of the predicted signal rate to the excluded value, $\left(\sigma B R_{\ell \nu}^{2}\right)_{I D M} /\left(\sigma B R_{\ell \nu}^{2}\right)_{95 \% \mathrm{CL}}$. The diagonal dashed line indicates $m_{H^{ \pm}}=m_{H}$ degeneracy. The exclusion extends all the way up to $m_{H^{+}} \simeq 90 \mathrm{GeV}$ for

\footnotetext{
${ }^{4}$ See also Refs. [2, 4].
} 


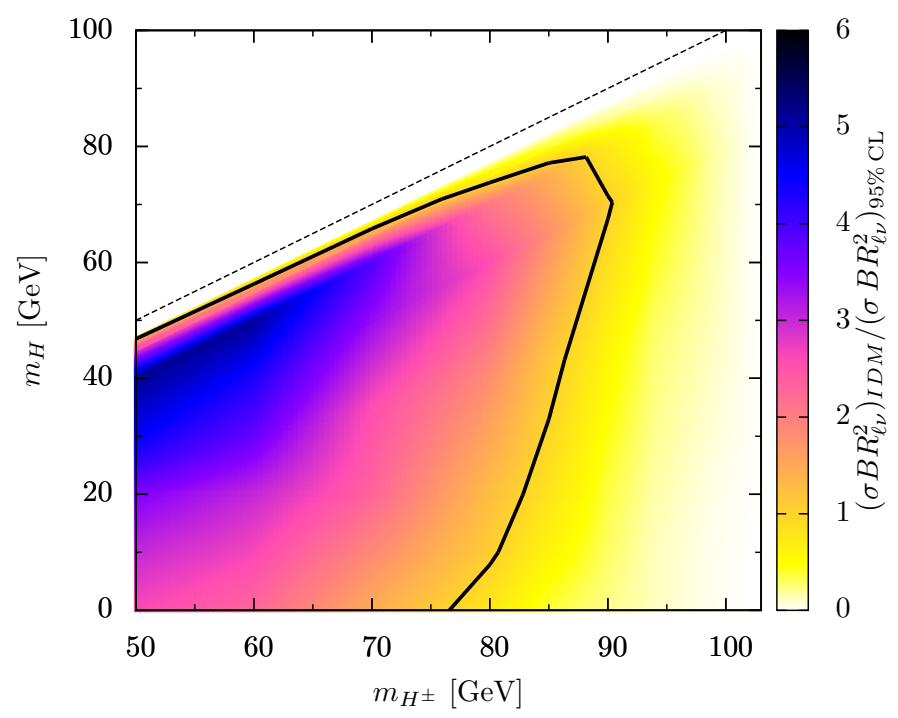

Figure 5: Excluded region (95\% CL) in the $m_{H^{ \pm}}-m_{H}$ plane derived from the OPAL search for leptons and missing energy of Ref. [65]. The colour map shows the magnitude of $\left(\sigma B R_{\ell \nu}^{2}\right)_{I D M} /\left(\sigma B R_{\ell \nu}^{2}\right)_{95 \% \mathrm{CL}}$

moderate mass splittings, in agreement with the analysis of Ref. [67], but falls off sharply in the degenerate limit of $\Delta^{ \pm} \lesssim 5 \mathrm{GeV}$ due to the reduced detection efficiencies for soft leptons. The IDM exclusion is also somewhat weaker than for charginos due to the lower relative production cross section.

It should be noted that our analysis assumes implicitly that the detection efficiencies are the same for the $\mathrm{H}^{+} H^{-}$signal as for $\chi_{1}^{+} \chi_{1}^{-}$when the charged and neutral masses match up. We expect this to be a good approximation based on the analysis of Ref. [31], which found that the efficiencies for a similar LEP search by the DELPHI collaboration for $\chi_{2}^{0} \chi_{1}^{0}$ production followed by $\chi_{2}^{0} \rightarrow \chi_{1}^{0} Z^{*}$ with $Z^{*} \rightarrow f \bar{f}$ were equal to those of the corresponding IDM process $A \rightarrow H Z^{*}$ with $Z^{*} \rightarrow f \bar{f}$ to within $10-20 \%$ throughout the relevant parameter space.

A full recasting [71] of the LEP analyses using the combined data from all four LEP experiments and including hadronic searches could provide a slightly stronger bound on the IDM mass spectrum. At the same time, the limits derived from such an analysis are unlikely to alter our general conclusions. Namely, we expect the compressed region of $\Delta^{ \pm} \lesssim 5 \mathrm{GeV}$ to remain unconstrained due to the difficulties in identifying objects with such small visible energies. To address this region, we turn next to LEP analyses designed to find charginos that are nearly degenerate with a lightest neutralino superpartner and apply them to the IDM. 


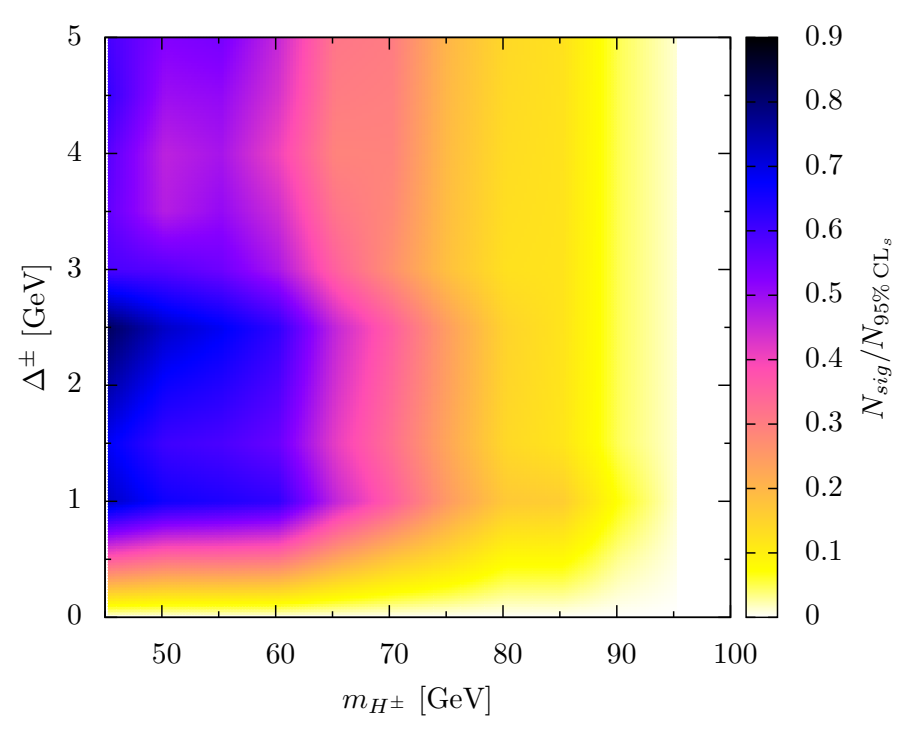

Figure 6: Magnitude of $\sigma\left(e^{+} e^{-} \rightarrow H^{+} H^{-} \gamma\right) / \sigma_{\mathrm{OPAL}}^{95 \%}$ C.L [75] in the $m_{H^{+}}-m_{H}$ plane.

\subsection{Compressed Searches for $H^{+} H^{-}$with a Photon}

Mass splittings below $\Delta^{ \pm} \lesssim 5 \mathrm{GeV}$ result in $H^{ \pm}$decays that leave very little hadronic or leptonic activity in the detector and that are difficult to distinguish from background. Some sensitivity can be regained by studying events with soft charged tracks together with an additional photon of moderate transverse momentum $p_{T}$ from initial- or final-state radiation. Searches of this type have been carried out in Refs. [68, 72, 73, 74, 75], and are summarized in Ref. [76]. In this section, we apply the OPAL analysis of Ref. [75] to the compressed IDM with charged mass splittings $\Delta^{ \pm} \lesssim 6 \mathrm{GeV}$.

The OPAL search of Ref. [75] was designed to probe nearly mass-degenerate charginos and neutralinos, and used $570 \mathrm{pb}^{-1}$ of data with center-of-mass energies between 189-208 GeV. Potential signal events were selected if they contained a high-energy photon with $E_{T}>5 \mathrm{GeV}$ and $\left|\cos \theta^{\gamma}\right|<0.976$ together with two to ten high quality tracks, with further cleaning and final selection cuts applied subsequently. To estimate the IDM signal in this search, we use Madgraph5 [70] to generate $H^{+} H^{-}$events in association with a photon having $E^{\gamma}>0.025 \sqrt{s}$ and $\left|\cos \theta^{\gamma}\right|>0.985$ from $e^{+} e^{-}$collisions at $\sqrt{s}=208 \mathrm{GeV}$. We then apply the efficiencies quoted in Ref. [75] for Higgsino-like charginos with the same photon requirements (Fig. 4c in Ref. [75]).5 To extrapolate these efficiencies to the entire $m_{H^{ \pm}}-\Delta^{ \pm}$plane, we assume a sharp linear rise from $\Delta^{ \pm}=0$ to $\Delta^{ \pm}=1 \mathrm{GeV}$ and a flat efficiency for $\Delta^{ \pm} \geq 1 \mathrm{GeV}$ (Fig. $4 \mathrm{~d}$ of Ref. [75]). As in the general chargino search considered above, our analysis assumes that the efficiencies for this subset of $H^{+} H^{-}$events will be very similar to the corresponding subset of chargino events. We expect this to be a reasonable approximation, but a full recasting would provide a more definitive answer.

With this estimate of the IDM signal, we attempt to derive limits on the theory in the

\footnotetext{
${ }^{5}$ Note that by applying the efficiencies to the subset of events with an additional hard photon, potential differences in the photon radiation spectrum between Higgsinos and the IDM scalars are taken into account.
} 
compressed regime by comparing our prediction to the number of observed and expected background events in the OPAL search (Fig. 3a,b of Ref. [75]). Applying a $\mathrm{CL}_{s}$ statistic, no limit is found at $95 \% \mathrm{CL}_{s}$. In Fig. 6 we show the ratio of the number of estimated $H^{+} H^{-}$ signal events to the upper limit allowed by the data, $N_{s i g} / N_{95 \% \mathrm{CL}_{s}}$, within the $m_{H^{ \pm}}-\Delta^{ \pm}$ plane. While no exclusion is found for $m_{H^{ \pm}}>m_{Z} / 2$, the signal does approach the exclusion limit for smaller $m_{H^{ \pm}}$masses. It is possible that an exclusion could be derived from a combination of data from all four LEP experiments. The combined limits given in Ref. [76] do not provide enough resolution to allow us to do so.

\subsection{Other LEP Searches}

A number of other LEP searches can be applied to the compressed IDM to probe both $H^{+} H^{-}$and $H A$ production. Very long-lived $H^{ \pm}$states will leave charged tracks in the LEP detectors. Searches for such tracks have been performed in Refs. [77, 78, 79], and typically require a track length of at least several $\mathrm{cm}$. The bounds derived from these searches are quite strict, approaching the kinematic production limit; the analysis of Ref. [79] excludes $H^{ \pm}$masses up to $m_{H^{ \pm}} \lesssim 95 \mathrm{GeV}$. However, lifetimes long enough to produce charged track lengths longer than a few centimetres only occur in the IDM for charged mass splittings below a few hundred $\mathrm{MeV}$ (Fig. 2), which is well below the typical expected splitting from quantum corrections (Fig. 1).

Various LEP searches can also be applied to $H A$ processes as in Ref. [31. In the moderately compressed regime, with neutral mass splittings in the range $\Delta^{0} \in[0.5,5] \mathrm{GeV}$, the OPAL monophoton plus charged tracks analysis of Ref. [75] applied to $\mathrm{H}^{+} \mathrm{H}^{-}$production above will likely have a similar sensitivity to $H A$ processes. Smaller mass splittings can be tested using pure monophoton searches (with a veto on charged tracks), and have been considered in Refs. [80, 81] and applied to simplified models of dark matter in Ref. [82]. We find that the cross sections excluded by these analyses are typically an order of magnitude larger than in the compressed IDM once the additional photon requirement is imposed.

In summary, the results of this section suggest that LEP does not constrain the compressed IDM for masses above $m_{Z} / 2$ provided the charged mass splitting lies below about $\Delta^{ \pm} \lesssim 5 \mathrm{GeV}$ and above a few hundred $\mathrm{MeV}$. We have also derived a refinement of the limits on $H^{+} H^{-}$production for $\Delta^{ \pm}$larger than $5 \mathrm{GeV}$.

\section{LHC Limits and Projected Sensitivity}

As we have seen, LEP searches can constrain the IDM up to masses close to $90 \mathrm{GeV}$, but they do not provide a bound beyond $m_{H}>m_{Z} / 2$ when the spectrum is compressed. In this section, we investigate whether existing and future searches at the LHC can further probe this region of the IDM. Motivated by the discussion of Sec. 2, we restrict our attention to the compressed scenario with $\Delta^{0}=\left(m_{A}-m_{H}\right) \sim 100 \mathrm{keV}-5 \mathrm{GeV} \ll \Delta^{ \pm}$and $\Delta^{ \pm}=$ $\left(m_{H^{ \pm}}-m_{H}\right) \sim 1-30 \mathrm{GeV}$ with $m_{H}>m_{Z} / 2$. Our general conclusion is that this regime is 
also very difficult to test at the LHC, but that mono-jet searches at $\sqrt{s}=14 \mathrm{TeV}$ could be sensitive to lower masses.

The dominant production modes of the $H, A$, and $H^{ \pm}$states at the LHC are

$$
\begin{aligned}
& q \bar{q} \rightarrow Z^{*} \rightarrow H A \\
& q \bar{q} \rightarrow \gamma / Z^{*} \rightarrow H^{+} H^{-} \\
& q \bar{q} \rightarrow W^{ \pm *} \rightarrow H^{ \pm} H, H^{ \pm} A \\
& g g \rightarrow h^{(*)} \rightarrow H H, A A, H^{+} H^{-}
\end{aligned}
$$

The first three processes are electroweak, while the last involves the additional difficulty of producing a Higgs. Production through the Higgs is also proportional to $\lambda_{L}^{2}$, and is suppressed even further when this coupling is small [83]. We will neglect it in the present discussion ${ }^{6}$ In this limit, the production of inert scalars is very similar to that of light degenerate Higgsinos in the MSSM, but with a rate that is smaller by about an order of magnitude.

\subsection{Exclusive Lepton Searches}

Several previous studies have analyzed leptonic signatures of the IDM at the LHC [2, 4, 5, 84, 85, 86]. Of the various final states considered, dilepton searches are typically the most promising. However, they are unlikely to provide additional constraints on the inert doublet model in the compressed limit. For example, Ref. [86] used the ATLAS study of Ref. [87] to constrain the IDM, considering events with exactly two leptons with $p_{T}>35,20 \mathrm{GeV}$. From these existing results, Ref. [86] found new limits for $\Delta^{0} \gtrsim 65 \mathrm{GeV}$. However, for smaller mass differences, no new limit is found due to the lepton $p_{T}$ requirements of the search.

More specific IDM searches using dileptons were proposed for the $14 \mathrm{TeV}$ LHC in Refs. 4, 5]. Both focus on the production channel of Eq. (30) and make use of the lepton kinematics expected from their origin in $Z^{*} \rightarrow \ell^{+} \ell^{-}$. Specifically, for small $\Delta^{0}$ one expects $m_{\ell \ell} \sim \Delta^{0}$, small lepton $p_{T}$, and nearly collinear leptons in most events; this helps to reduce the SM background. The parton-level result of Ref. [4] finds $S / \sqrt{B} \sim 5$ for $100 \mathrm{fb}^{-1}$ of data (with $S / B \sim 1)$ for $\left(m_{H}, m_{A}\right)=(50,60) \mathrm{GeV}$. However, Ref. [5] performed a similar analysis and found less optimistic results once lepton isolation and detector effects were taken into account. It is possible that a lepton-jet type search could recover some sensitivity [88], but the compressed limit of the IDM is unlikely to be covered by these strategies.

Searches with trileptons were considered in Ref. 84] while four-lepton signatures were studied in Ref. [85]. In both cases, no limits appear to be attainable at the LHC for small mass splittings, even at $14 \mathrm{TeV}$ and large integrated luminosity.

\footnotetext{
${ }^{6}$ Small $\left|\lambda_{L}\right|$ is also motivated by the direct detection bounds found in Sec. 4 . Note as well that for small $\left|\lambda_{L}\right|$, modifications to the production and decay rates of the SM Higgs boson $h$ will also be negligible.
} 


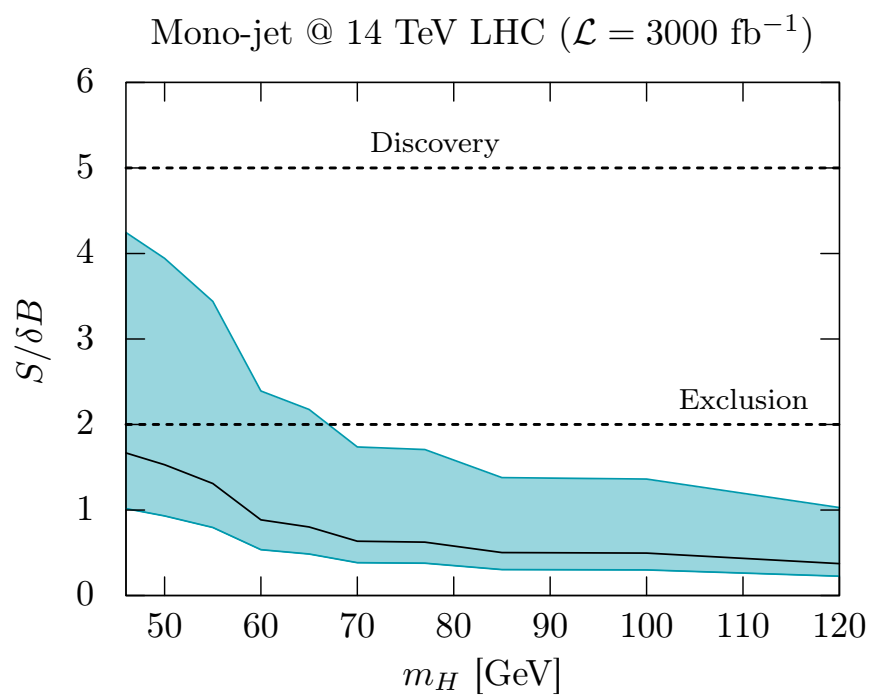

Figure 7: Predicted signal significance in the mono-jet channel at the $14 \mathrm{TeV}$ LHC with integrated luminosity $\mathcal{L}=3000 \mathrm{fb}^{-1}$ as a function of $m_{H}$ for the specific set of cuts described in Appendix A . The solid black line corresponds to a background systematic uncertainty $\epsilon_{\mathrm{bkg}}$ of $3 \%$. The shaded band was obtained by varying $\epsilon_{\mathrm{bkg}}$ from $1-5 \%$.

\subsection{Mono-Jet Searches}

Dedicated lepton searches decrease in sensitivity in the degenerate regime simply because the resulting leptons are very soft. This suggests an alternative detection strategy for the inert scalars of the compressed IDM: search for a hard object (e.g. a jet, Higgs, or gauge boson) produced in association with substantial missing energy. For the models of interest, the pure mono-jet channel is the most promising. Searches of this type typically veto on leptons with $p_{T}>p_{T}^{\ell, \min } \gtrsim 10 \mathrm{GeV}$. If both $\Delta^{ \pm}<p_{T}^{\ell, \min }$ and $\Delta^{0}<2 p_{T}^{\ell, \min }$, the transverse momentum carried away by all three inert states $\left(H, A, H^{ \pm}\right)$can be counted as missing energy $\left(\mathbb{E}_{T}\right)$. In what follows, we assume both splittings are below $p_{T}^{\ell, \min }$ to show the maximal LHC sensitivity to the compressed limit through these searches. For our Monte Carlo analyses, we therefore set $m_{H}=m_{A}=m_{H^{ \pm}}$for simplicity.

Current ATLAS and CMS mono-jet searches using $8 \mathrm{TeV}$ data do not constrain the compressed IDM for $m_{H}>m_{Z} / 2$. We have verified this through a parton-level analysis considering the limits on $\sigma \times \mathcal{A} \times \epsilon$ from the ATLAS search in Ref. [89], where $\mathcal{A}$ is the signal acceptance and $\epsilon$ the efficiency. Events are simulated in MadGraph5 [70] and analyzed using the selection criteria outlined in Ref. [89], assuming $\epsilon=1$ for the signal. Considering the nine signal regions defined in Ref. [89], we find $\sigma \times \mathcal{A} \times \epsilon /(\sigma \times \mathcal{A} \times \epsilon)_{95 \% \mathrm{CL}_{\mathrm{s}}}<0.5$ for all values of $m_{H}>m_{Z} / 2$. Although this analysis is done at parton level, we do not expect showering or detector effects to make a qualitatively significant difference, due to the various vetoes on additional objects besides the hard jet and missing energy. We have explicitly verified this to be the case for the $14 \mathrm{TeV}$ projections discussed below. 
Mono-jet searches at the $14 \mathrm{TeV}$ LHC may begin to test the compressed IDM with sufficiently high integrated luminosity provided systematic uncertainties on the backgrounds can be tightly controlled. To estimate the sensitivity of these searches, signal and background events are generated in Madgraph5 [70], showered in Pythia6 [90], and processed in Delphes3 91] for fast detector simulation. We then impose the cuts and selection criteria described in Appendix A. The results of this analysis are shown in in Fig. 7, where we plot the predicted mono-jet signal significance at $14 \mathrm{TeV}$ with $\mathcal{L}=3000 \mathrm{fb}^{-1}$ of data as a function of the mass $m_{H}$. The significance shown in this figure is defined to be

$$
\frac{S}{\delta B} \equiv \frac{S}{\sqrt{B+\epsilon_{\mathrm{bkg}}^{2} B^{2}+\epsilon_{\mathrm{sig}}^{2} S^{2}}},
$$

where $S$ is the signal, $B$ is the background, and $\epsilon_{\text {sig }}$ and $\epsilon_{\mathrm{bkg}}$ are their uncertainties.

Our simulations were performed at leading order including up to two hard jets at the parton level. We do not expect a full next-to-leading order (NLO) calculation to significantly alter our results. The largest background contributions are from $p p \rightarrow Z j(+X) \rightarrow \nu \bar{\nu} j(+X)$, and $p p \rightarrow W^{ \pm} j(+X) \rightarrow \ell \nu j(+X)$, which involve the same diagrams as the dominant signal contributions with the neutrinos and charged leptons replacing the inert scalars in the final state (see Eqs. 30 31). We therefore expect NLO corrections to affect the signal and background distributions similarly. Moreover, the most significant NLO QCD corrections to the background are from the emission of an additional hard jet [92], which we account for by considering up to one additional jet at the parton level. Our minimum $p_{T}$ cut on the second jet eliminates collinear enhancements in the cross-section, and so we expect virtual NLO effects to be relatively small.

The shaded region in Fig. 7 is the result of varying the systematic uncertainty $\epsilon_{\mathrm{bkg}}$ in the range $[0.01,0.05]$, with the solid black line corresponding to $\epsilon_{\mathrm{bkg}}=0.03$. This range corresponds to that suggested by ATLAS for 14 TeV LHC mono-jet searches in Ref. [93]. The background systematics are dominated by uncertainties in the absolute jet energy scale and resolution, in Monte Carlo simulations, and in the determination of lepton momenta. It is difficult to predict the evolution of systematic uncertainties over the course of an experiment. However, we expect the range shown to approximate both the near- and long-term prospects for the $14 \mathrm{TeV}$ LHC, in line with the ATLAS analysis in Ref. [93. The signal systematic uncertainty was set to $\epsilon_{\mathrm{sig}}=0.1$, following the analysis of Ref. 94] for compressed Higgsinos.

All points considered in Fig. 7 feature at least 10 signal events. We also perform an analogous parton-level analysis and find that it reproduces the full result for $S / \delta B$ within $10-15 \%$ across the entire mass range considered.

The results shown in Fig. 7 suggest that the $14 \mathrm{TeV}$ LHC can probe masses up to about $70 \mathrm{GeV}$, provided background systematic uncertainties are controlled to within 1\%. Such small uncertainties are likely unrealistic [95, 96], although they may be possible in the longterm [93]. If the background systematics are larger than about $2 \%$, this search will not be able to exclude any of the $m_{H}>m_{Z} / 2$ region at the $14 \mathrm{TeV}$ LHC. It is possible that a harder missing energy cut could provide slightly more sensitivity. However, the background systematics are likely to increase substantially as one moves farther out on the high- $\mathbb{E}_{T}$ tail. 
Some improvement in sensitivity over standard mono-jet searches might be obtained by demanding additional soft leptons in the event from $H^{ \pm}$or $A$ decays. This technique has shown promise for probing compressed electroweakinos [94, 97, 98, 99, 100, 101, 102] and sleptons [103, 104] in supersymmetric models. However, comparing the compressed IDM to scenarios with degenerate Higgsinos, this strategy does not appear to help very much. In the IDM, we are primarily interested in small $\Delta^{0}$ and larger $\Delta^{ \pm}$. For Higgsinos, the asymptotic decoupling limit is $\Delta^{0} \simeq 2 \Delta^{ \pm}[100$, 102]. The most promising compressed Higgsino searches therefore focus on $\chi_{2}^{0} \rightarrow \chi_{1}^{0} Z^{*}$ decays with $Z^{*} \rightarrow \ell \ell$ since the leptons are likely to be more energetic, but also because the correlated kinematics between them allows for a good discrimination from background. In the compressed IDM with small $\Delta^{0}$, the observable leptons will come primarily from $H^{+} \rightarrow H W^{*}$ with $W^{*} \rightarrow \ell \nu_{\ell}$, and so are less likely to pass the soft-lepton kinematic selection criteria in these searches [100, 102]. For very small neutral mass splittings the $A \rightarrow H Z^{*}$ decay can be displaced, and searches for a mono-jet with a soft displaced vertex are also possible, as suggested for models of inelastic DM in Refs. [105, 106, 107]. In the IDM, we find that macroscopic decay lengths $c \tau>1 \mathrm{~mm}$ only occur for $\Delta^{0} \lesssim 1 \mathrm{GeV}$, and thus the displaced decay products of the $A \rightarrow H Z^{*}$ decays are typically too soft to be identified by the LHC detectors.

Taken together, our results suggest that the LHC will be unlikely to probe the compressed IDM much above $m_{Z} / 2$, except perhaps in the mono-jet channel at $14 \mathrm{TeV}$ provided provided background systematics can be greatly suppressed. For this reason, we turn next to investigate the sensitivity of future colliders to this scenario.

\section{$7 \quad$ Future Colliders}

To conclude our study, we consider the prospects for testing the compressed IDM at a future $100 \mathrm{TeV} p p$ collider and an ILC-type $e^{+} e^{-}$collider.

\subsection{TeV pp Collider}

A $100 \mathrm{TeV}$ proton-proton collider is likely to significantly surpass the current LHC sensitivity to electroweakinos [94, 108, 109, 110, 111], and so it is worthwhile to consider its possible impact on the compressed IDM. To do so, we use Madgraph5 [70] to perform a parton-level analysis of searches with a hard jet and large missing energy, as described in Appendix A. We expect that including showering and detector effects will not significantly alter the results. This was true of our 14 TeV LHC mono-jet study. Note also that no detector design currently exists for a $100 \mathrm{TeV}$ collider.

The results of our analysis are shown in Fig. 8 for a representative set of cuts and selection criteria detailed in Appendix A. The shaded region in this figure corresponds to varying the systematic uncertainty on the background $\epsilon_{\mathrm{bkg}}$ between $1 \%-5 \%$. As expected, the reach is substantially higher than that of the $14 \mathrm{TeV}$ LHC. However, masses above around $200 \mathrm{GeV}$ will likely be difficult to probe. Comparing these results to those of Ref. [94], which studied 


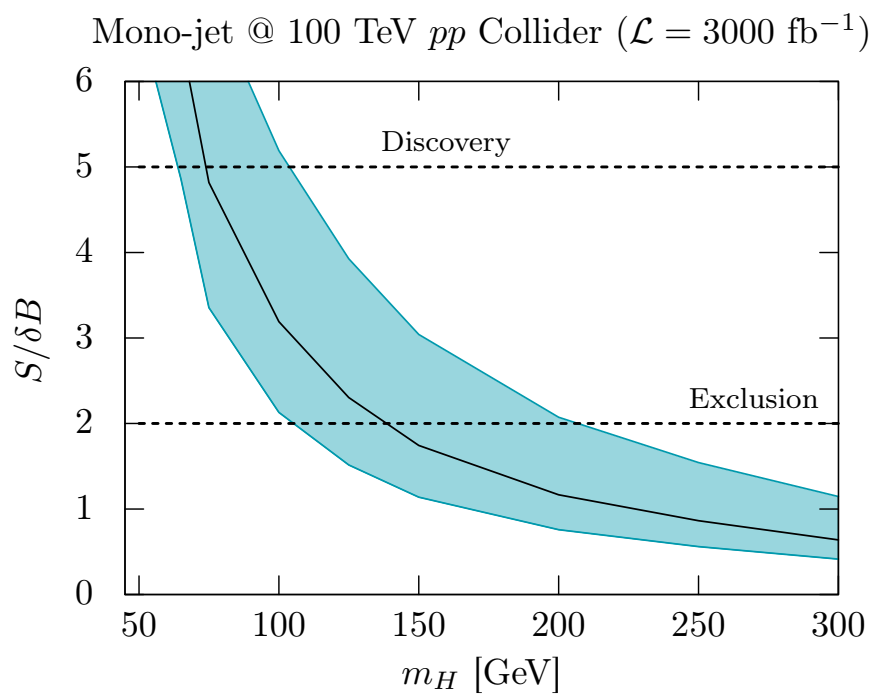

Figure 8: Estimated signal significance in the mono-jet channel at a $100 \mathrm{TeV} p p$ collider as a function of $m_{H}$ for a particular set of cuts, detailed in Appendix A. The black line corresponds to a background systematic error $\epsilon_{\mathrm{bkg}}=3 \%$, while the shaded band was obtained by varying $\epsilon_{\mathrm{bkg}}$ from $1-5 \%$.

compressed Higgsinos at a $100 \mathrm{TeV}$ collider, the reach we find is about a factor of four lower in mass due to the smaller production cross-section in the IDM.

Mono-jet searches requiring additional soft leptons or disappearing tracks were also shown to be promising for compressed Higgsinos in Ref. [94]. The former is unlikely to significantly improve on the pure mono-jet search in the compressed IDM for the reasons discussed in Sec. 6.2. Meanwhile, disappearing track searches are very sensitive to the chargino lifetime, which is governed by the chargino-neutralino mass splitting. For a pure Higgsino, this splitting is about $\Delta^{ \pm} \simeq 355 \mathrm{MeV}\left[112\right.$. Since we generally expect larger values of $\Delta^{ \pm}$in the IDM, these disappearing track searches will not typically be applicable. We therefore expect that Fig. 8 represents a reasonable sensitivity estimate for a future $100 \mathrm{TeV} p p$ collider, given the information currently available about such a facility.

\subsection{High-energy Lepton Collider}

A high-energy $e^{+} e^{-}$ILC-type collider would provide an additional tool to probe the compressed IDM. The clean environment of such a machine, together with the option for polarized beams and low-threshold detectors, is well-suited for this scenario.

To estimate the reach of a potential ILC for the compressed IDM, we reinterpret the analysis of Ref. [113] which studied a light "slepton" scenario consisting of a $(2,-1 / 2)$ electroweak scalar doublet $(\widetilde{\nu}, \widetilde{\ell})$ with only direct couplings to the electroweak vector bosons of the SM. A tree-level ( $D$-term) mass splitting is assumed to push the charged state to 
be slightly heavier such that it decays promptly to the lighter state through an off-shell $W$ boson. The analysis of Ref. [113] demands a single hard photon and large missing energy, and finds an exclusion $(2 \sigma)$ reach of about $140 \mathrm{GeV}(160 \mathrm{GeV})$ for $\gamma \widetilde{\nu} \widetilde{\nu}^{*}\left(\gamma \widetilde{\ell} \ell^{*}\right)$ production with $500 \mathrm{fb}^{-1}$ of data at $\sqrt{s}=500 \mathrm{GeV}$ and making use of polarized beams to reduce the dominant background from $e^{+} e^{-} \rightarrow \gamma \nu \bar{\nu}$. Considerably stronger bounds are found for light degenerate Higgsinos due once again to the lower net production rates for scalars and the faster turn off of their production cross-sections as one approaches the kinematic threshold.

The "slepton" model studied in Ref. [113] can be mapped directly onto the compressed IDM with $\widetilde{\nu} \leftrightarrow(H+i A) / \sqrt{2}$ and $\widetilde{\ell} \leftrightarrow H^{-}$, which have the same gauge quantum numbers. The limits of this analysis are directly applicable to $H A$ and $H^{+} H^{-}$production in the very compressed limit of $\Delta^{0, \pm} \lesssim 1 \mathrm{GeV}$. However, since no attempt is made to distinguish between the charged $(\widetilde{\ell})$ and neutral $(\widetilde{\nu})$ states, and no veto on additional objects is imposed, we expect that these bounds will not be degraded too severely for larger mass splittings up to the roughly $\Delta \lesssim 10 \mathrm{GeV}$ we are most interested in.

Based on the results of our LEP analyses, the ILC sensitivity to the compressed IDM with $\Delta^{ \pm} \gtrsim 3 \mathrm{GeV}$ can likely be improved further relative to the pure monophoton analysis of Ref. 113 by searching for soft leptons in addition to a hard photon and missing energy. This strategy has been applied to nearly-degenerate Higgsinos in the ILC studies of Refs. [114, 115, 116, 117. Since an ILC-type detector is expected to have a very low lepton $p_{T}$ threshold, perhaps approaching $0.5 \mathrm{GeV}$ [118], it might even be possible to improve the sensitivity for even smaller mass splittings. It would be interesting to revisit this possibility once the sensitivities of an ILC detector are better known. For larger mass splittings $\Delta^{ \pm} \gtrsim 10 \mathrm{GeV}$, the mono-photon requirement can be dropped and searches can be performed for leptons (or jets) and missing energy as described in Refs. [119, 120].

\section{Conclusions}

In this study, we have performed a detailed analysis of the inert doublet model in the compressed limit. Mass degeneracies can arise in the presence of approximate continuous symmetries. The compressed scenario allows for light electroweakly-charged scalars that are compatible with electroweak precision observables, results from dark matter experiments, and cosmological constraints. Unfortunately, these new states can be difficult to test at colliders.

We have carefully analyzed the constraints from LEP on the compressed IDM. A reinterpretation of the OPAL dilepton plus MET search excludes masses up to about $90 \mathrm{GeV}$ for charged-neutral mass splittings above $5 \mathrm{GeV}$. However, there are currently no direct constraints on masses above $m_{Z} / 2$ for charged-neutral mass splittings less than $5 \mathrm{GeV}$. These conclusions are a refinement on past work, which had previously focused on mass splittings above several GeV.

Current $8 \mathrm{TeV}$ LHC results yield no additional constraints beyond LEP in the compressed region. Leptons are typically too soft to trigger on in exclusive lepton searches, and the mono- 
jet production cross-sections are significantly smaller than those for Higgsinos. However, the $14 \mathrm{TeV}$ LHC may begin to probe low masses in the mono-jet channel, although this depends quite sensitively on the systematic uncertainties for the backgrounds. Our results suggest that uncertainties below $\sim 2-3 \%$ may be sufficient to exclude masses only slightly above $m_{Z} / 2$ with $3000 \mathrm{fb}^{-1}$ of data. We also do not expect searches for mono-jet topologies with additional soft decay products, charged tracks, or displaced vertices to significantly alter this conclusion.

Future colliders can offer considerably better opportunities for testing the compressed IDM. A $100 \mathrm{TeV} p p$ may be able to probe inert doublet masses up to $100-200 \mathrm{GeV}$ in the monojet channel, with the precise limit depending on the degree to which systematic uncertainties can be controlled (with a range of 1-5\% assumed here). For an ILC-like $e^{+} e^{-}$collider with center-of-mass energy $\sqrt{s}=500 \mathrm{GeV}$ and $500 \mathrm{fb}^{-1}$ of data, we find a sensitivity up to masses of $m=140 \mathrm{GeV}(160 \mathrm{GeV})$ for $H A\left(H^{+} H^{-}\right)$in the highly compressed limit using a pure mono-photon search. An even larger reach is expected away from the compressed limit by including leptons in the search channels. Ultimately, however, these future experiments are unlikely to probe the region of the compressed IDM in which the lightest inert scalar saturates the observed dark matter abundance via thermal freezeout. In this high-mass region, direct detection efforts are likely to offer the most promise. Nevertheless, a combination of LHC, ILC, and $100 \mathrm{TeV}$ collider searches would be valuable in conclusively testing low- to intermediate-mass scalars in the compressed inert doublet model.

\section{Acknowledgments}

We thank Eder Izaguirre, Robert McPherson, Brian Shuve, and Isabel Trigger for helpful discussions. This work is supported by the Natural Sciences and Engineering Research Council of Canada (NSERC).

\section{A Mono-jet Projections}

In this appendix, we detail our mono-jet analysis of the compressed IDM.

\section{A.1 14 TeV LHC}

We perform our $14 \mathrm{TeV}$ LHC analysis at the detector level, using MadGraph5 [70] to generate events, Pythia6 [90] to shower and hadronize them, and Delphes3 [91] as a fast detector simulator. Our strategy is similar to that found in previous mono-jet studies [94, 96, 101, 93. Specifically, we select events if they satisfy the following criteria:

$$
\begin{gathered}
p_{T}^{j_{1}}>300 \mathrm{GeV},\left|\eta_{j_{1}}\right|<2.0, \mathbb{E}_{T}>1 \mathrm{TeV}, \\
\Delta \phi\left(j_{1,2}, \mathbb{E}_{T}\right)>0.5, p_{T}^{j_{2}}<100 \mathrm{GeV}, N_{j} \leq 2
\end{gathered}
$$


where $j_{1}$ is the jet with the largest $p_{T}$, and identified jets are required to have $p_{T}>50 \mathrm{GeV}$ and $|\eta|<3.6$. Note that these jet definitions were suggested by ATLAS in Ref. [93] for studies of $14 \mathrm{TeV}$ mono-jet searches. Up to one additional identified jet is allowed; however we require $p_{T}^{j_{2}}<100 \mathrm{GeV}$, since the background $p_{T}^{j_{2}}$ distribution peaks at slightly higher values than the signal for low $m_{H}$ (see the bottom left panel of Fig. 9). We also include the following lepton vetoes:

- Veto on $e$ with $p_{T}(e)>7 \mathrm{GeV},|\eta(e)|<2.5$

- Veto on $\mu$ with $p_{T}(\mu)>7 \mathrm{GeV},|\eta(\mu)|<2.47$

- Veto on hadronic taus, with $p_{T}\left(\tau_{h}\right)>20 \mathrm{GeV},\left|\eta\left(\tau_{h}\right)\right|<2.3$.

We use the default CMS detector card implemented in Delphes3 and neglect pile-up effects. In addition to the cuts shown above, we consider several different values of $p_{T}^{j_{1}, \min }, p_{T}^{j_{2}, \min }$ as suggested in Refs. [94, 96, 101] for Higgsinos, and we vary $\mathbb{E}_{T}^{\min } \in[400,1400] \mathrm{GeV}$. The final choices listed represent one of the more promising configurations. Tighter MET cuts may offer slightly more sensitivity, however we expect the uncertainties in both the background and signal to increase quickly as $\mathbb{E}_{T}^{\text {min }}$ is raised.

The dominant backgrounds are $Z+$ jets, where $Z \rightarrow \bar{\nu} \nu$, and $W+$ jets where the $W$ decays leptonically. We validate our backgrounds by checking against the results of Refs. [96, 101]. The distributions of the various background events in $\mathbb{E}_{T}$ and $p_{T}^{j_{1}}$ before cuts are shown in Fig. 9, along with the signal for two different masses. The distribution of signal events closely resembles that of the backgrounds for large $\mathbb{E}_{T}$ and $p_{T}^{j_{1}}$, making discrimination difficult. Note that, after cuts, the $Z+$ jets background dominates in the signal region defined above.

\section{A.2 $100 \mathrm{TeV} p p$ Collider}

We perform our $100 \mathrm{TeV}$ analysis at parton level, since no detector design currently exists for such a collider. Signal and background events are generated in Madgraph5, and we neglect pile-up effects. The dominant backgrounds in this case are once again $Z+$ jets, where $Z \rightarrow \bar{\nu} \nu$, and $W+$ jets where the $W$ decays leptonically. We once again neglect the effect of pile-up. Our selection criteria are similar to those used for our LHC analysis, with higher cuts on the various transverse momenta. Specifically, we require:

$$
\begin{gathered}
p_{T}^{j_{1}}>1.2 \mathrm{TeV},\left|\eta_{j_{1}}\right|<2.0, \mathbb{E}_{T}>5 \mathrm{TeV}, \\
\Delta \phi\left(j_{1,2}, \mathbb{E}_{T}\right)>0.5, \quad N_{j} \leq 2
\end{gathered}
$$

where identified jets are required to have $p_{T}>250 \mathrm{GeV}$ and $|\eta|<3.6$. We do not include an upper limit on $p_{T}^{j_{2}}$, as we find it has little effect on the distributions in this case. Following Ref. [94, the lepton vetoes are chosen as follows:

- Veto on $e$ with $p_{T}(e)>20 \mathrm{GeV},|\eta(e)|<2.5$ 

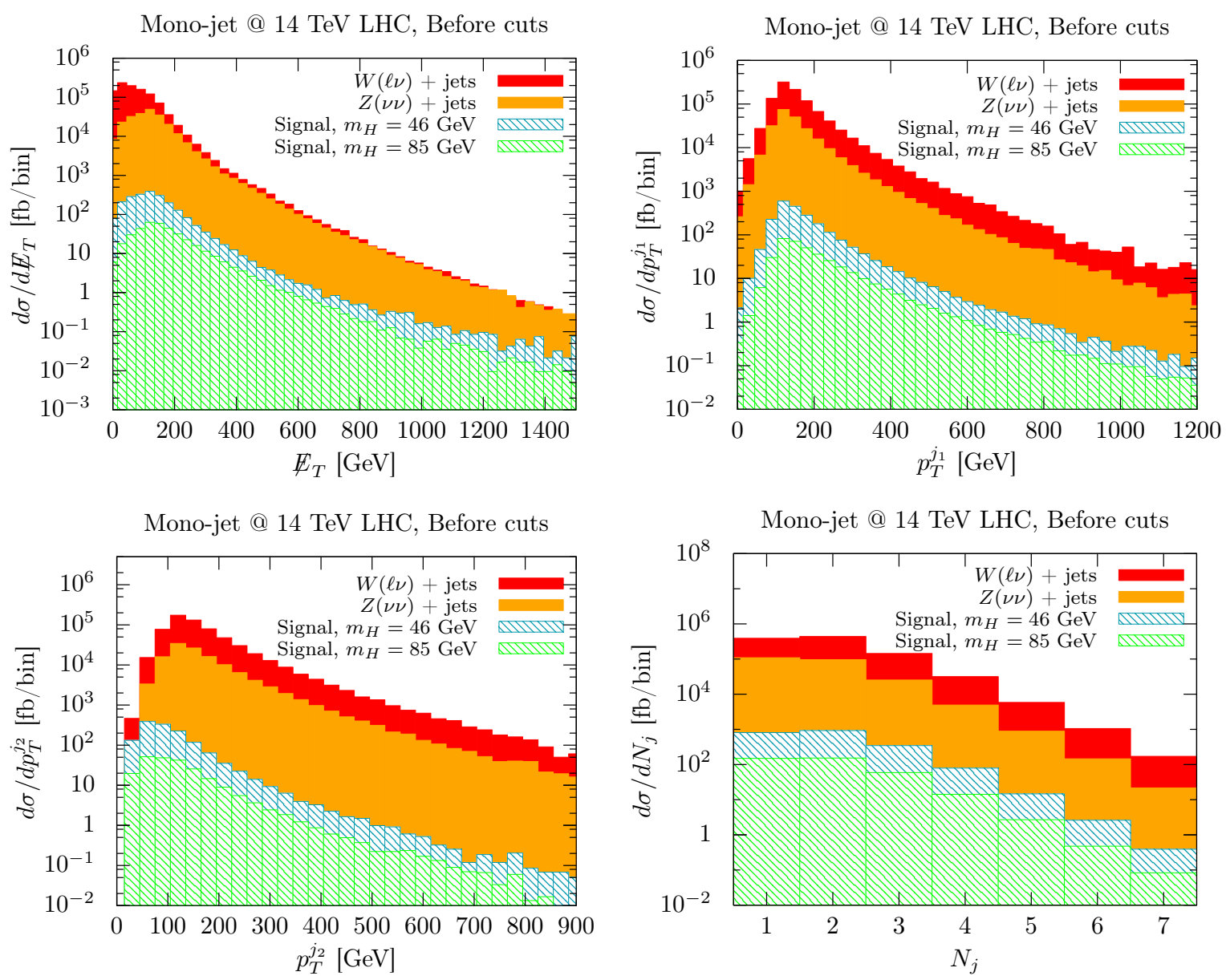

Figure 9: Sample histograms of the signal and background events generated for our $14 \mathrm{TeV}$ LHC mono-jet analysis before cuts. The shapes of all the signal distributions mimic those of the background, making discrimination from the background difficult. Note that, after cuts, the $Z$ background dominates the signal regions we consider.

- Veto on $\mu$ with $p_{T}(\mu)>20 \mathrm{GeV},|\eta(\mu)|<2.1$

- Veto on hadronic taus, with $p_{T}\left(\tau_{h}\right)>40 \mathrm{GeV},\left|\eta\left(\tau_{h}\right)\right|<2.3$.

We investigate the effect of varying the cut on missing transverse energy between 2.5-7.0 $\mathrm{TeV}$. As in the LHC analysis, tighter MET cuts may slightly improve the reach, especially for cases with larger $\epsilon_{\mathrm{bkg}}$, however once again the systematic uncertainties in the signal and backgrounds are expected to increase significantly for larger $\mathbb{E}_{T}^{\min }$. Note that Ref. 94] used similar cuts for studying compressed Higgsinos at a $100 \mathrm{TeV}$ collider. 


\section{References}

[1] N. G. Deshpande and E. Ma, Phys. Rev. D 18, 2574 (1978).

[2] R. Barbieri, L. J. Hall and V. S. Rychkov, Phys. Rev. D 74, 015007 (2006) [hep$\mathrm{ph} / 0603188]$.

[3] L. Lopez Honorez, E. Nezri, J. F. Oliver and M. H. G. Tytgat, JCAP 0702, 028 (2007) [hep-ph/0612275].

[4] Q. H. Cao, E. Ma and G. Rajasekaran, Phys. Rev. D 76, 095011 (2007) [arXiv:0708.2939 [hep-ph]].

[5] E. Dolle, X. Miao, S. Su and B. Thomas, Phys. Rev. D 81, 035003 (2010) [arXiv:0909.3094 [hep-ph]].

[6] M. Hirsch, S. Morisi, E. Peinado and J. W. F. Valle, Phys. Rev. D 82, 116003 (2010) [arXiv:1007.0871 [hep-ph]].

[7] M. S. Boucenna, M. Hirsch, S. Morisi, E. Peinado, M. Taoso and J. W. F. Valle, JHEP 1105, 037 (2011) [arXiv:1101.2874 [hep-ph]].

[8] T. Brown, C. Frugiuele and T. Gregoire, JHEP 1106, 108 (2011) [arXiv:1012.2060 [hep$\mathrm{ph}]$.

[9] L. Lavoura, S. Morisi and J. W. F. Valle, JHEP 1302, 118 (2013) [arXiv:1205.3442 [hep-ph]].

[10] T. A. W. Martin and A. de la Puente, Phys. Lett. B 727, 443 (2013) [arXiv:1304.7835 [hep-ph]].

[11] T. W. Kephart and T. C. Yuan, arXiv:1508.00673 [hep-ph].

[12] N. Nagata, K. A. Olive and J. Zheng, JHEP 1510, 193 (2015) [arXiv:1509.00809 [hep$\mathrm{ph}]$.

[13] A. Carmona and M. Chala, JHEP 1506, 105 (2015) [arXiv:1504.00332 [hep-ph]].

[14] T. A. Chowdhury, M. Nemevsek, G. Senjanovic and Y. Zhang, JCAP 1202, 029 (2012) [arXiv:1110.5334 [hep-ph]].

[15] D. Borah and J. M. Cline, Phys. Rev. D 86, 055001 (2012) [arXiv:1204.4722 [hep-ph]].

[16] G. Gil, P. Chankowski and M. Krawczyk, Phys. Lett. B 717, 396 (2012) [arXiv:1207.0084 [hep-ph]].

[17] S. S. AbdusSalam and T. A. Chowdhury, JCAP 1405, 026 (2014) [arXiv:1310.8152 [hep-ph]]. 
[18] N. Blinov, J. Kozaczuk, D. E. Morrissey and C. Tamarit, Phys. Rev. D 92, no. 3, 035012 (2015) [arXiv:1504.05195 [hep-ph]].

[19] N. Blinov, S. Profumo and T. Stefaniak, JCAP 1507, no. 07, 028 (2015) [arXiv:1504.05949 [hep-ph]].

[20] T. Hambye, F.-S. Ling, L. Lopez Honorez and J. Rocher, JHEP 0907, 090 (2009) [JHEP 1005, 066 (2010)] [arXiv:0903.4010 [hep-ph]].

[21] J.-M. Gerard and M. Herquet, Phys. Rev. Lett. 98, 251802 (2007) [hep-ph/0703051 [HEP-PH]].

[22] A. Goudelis, B. Herrmann and O. Stål, JHEP 1309, 106 (2013) [arXiv:1303.3010 [hep$\mathrm{ph}]$.

[23] M. Cirelli, N. Fornengo and A. Strumia, Nucl. Phys. B 753, 178 (2006) [hep$\mathrm{ph} / 0512090]$.

[24] J. H. Kuhn and A. Santamaria, Z. Phys. C 48, 445 (1990).

[25] C. H. Chen, M. Drees and J. F. Gunion, Phys. Rev. Lett. 76, 2002 (1996) [hep$\mathrm{ph} / 9512230]$.

[26] C. H. Chen, M. Drees and J. F. Gunion, Phys. Rev. D 55, 330 (1997) [Phys. Rev. D 60, 039901 (1999)] [hep-ph/9607421].

[27] C. H. Chen, M. Drees and J. F. Gunion, hep-ph/9902309.

[28] J. F. Donoghue, E. Golowich and B. R. Holstein, "Dynamics of the standard model," Camb. Monogr. Part. Phys. Nucl. Phys. Cosmol. 2, 1 (1992).

[29] H. E. Haber and D. Wyler, Nucl. Phys. B 323, 267 (1989).

[30] J. Bramante, A. Delgado, F. Elahi, A. Martin and B. Ostdiek, Phys. Rev. D 90, no. 9, 095008 (2014) [arXiv:1408.6530 [hep-ph]].

[31] E. Lundstrom, M. Gustafsson and J. Edsjo, Phys. Rev. D 79, 035013 (2009) [arXiv:0810.3924 [hep-ph]].

[32] K. A. Olive et al. [Particle Data Group Collaboration], Chin. Phys. C 38, 090001 (2014).

[33] G. Belanger, B. Dumont, U. Ellwanger, J. F. Gunion and S. Kraml, Phys. Rev. D 88, 075008 (2013) [arXiv:1306.2941 [hep-ph]].

[34] A. Arhrib, R. Benbrik and N. Gaur, Phys. Rev. D 85, 095021 (2012) [arXiv:1201.2644 [hep-ph]].

[35] B. Swiezewska and M. Krawczyk, Phys. Rev. D 88, no. 3, 035019 (2013) [arXiv:1212.4100 [hep-ph]].

[36] E. M. Dolle and S. Su, Phys. Rev. D 80, 055012 (2009) [arXiv:0906.1609 [hep-ph]]. 
[37] L. Lopez Honorez and C. E. Yaguna, JHEP 1009, 046 (2010) [arXiv:1003.3125 [hep-ph]].

[38] L. Lopez Honorez and C. E. Yaguna, JCAP 1101, 002 (2011) [arXiv:1011.1411 [hep-ph]].

[39] M. Krawczyk, D. Sokolowska, P. Swaczyna and B. Swiezewska, JHEP 1309, 055 (2013) [arXiv:1305.6266 [hep-ph]].

[40] A. Arhrib, Y. L. S. Tsai, Q. Yuan and T. C. Yuan, JCAP 1406, 030 (2014) [arXiv:1310.0358 [hep-ph]].

[41] A. Ilnicka, M. Krawczyk and T. Robens, arXiv:1508.01671 [hep-ph].

[42] P. A. R. Ade et al. [Planck Collaboration], arXiv:1502.01589 [astro-ph.CO].

[43] G. Belanger, F. Boudjema, A. Pukhov and A. Semenov, Comput. Phys. Commun. 185, 960 (2014) [arXiv:1305.0237 [hep-ph]].

[44] B. W. Lee, C. Quigg and H. B. Thacker, Phys. Rev. D 16, 1519 (1977).

[45] M. S. Chanowitz and M. K. Gaillard, Nucl. Phys. B 261, 379 (1985).

[46] D. Tucker-Smith and N. Weiner, Phys. Rev. D 64, 043502 (2001) [hep-ph/0101138].

[47] C. Arina, F. S. Ling and M. H. G. Tytgat, JCAP 0910, 018 (2009) [arXiv:0907.0430 [hep-ph]].

[48] D. Majumdar and A. Ghosal, Mod. Phys. Lett. A 23, 2011 (2008) doi:10.1142/S0217732308025954 [hep-ph/0607067].

[49] S. Kanemura, S. Matsumoto, T. Nabeshima and N. Okada, Phys. Rev. D 82, 055026 (2010) [arXiv:1005.5651 [hep-ph]].

[50] R. J. Hill and M. P. Solon, Phys. Rev. D 91, 043505 (2015) [arXiv:1409.8290 [hep-ph]].

[51] D. S. Akerib et al. [LUX Collaboration], arXiv:1512.03506 [astro-ph.CO].

[52] R. Agnese et al. [SuperCDMS Collaboration], [arXiv:1509.02448 [astro-ph.CO]].

[53] G. Angloher et al., Astropart. Phys. 18, 43 (2002).

[54] D. S. Akerib et al. [LZ Collaboration], arXiv:1509.02910 [physics.ins-det].

[55] P. Cushman et al., arXiv:1310.8327 [hep-ex].

[56] M. Gustafsson, E. Lundstrom, L. Bergstrom and J. Edsjo, Phys. Rev. Lett. 99, 041301 (2007) [astro-ph/0703512 [ASTRO-PH]].

[57] P. Agrawal, E. M. Dolle and C. A. Krenke, Phys. Rev. D 79, 015015 (2009) [arXiv:0811.1798 [hep-ph]].

[58] S. Andreas, M. H. G. Tytgat and Q. Swillens, JCAP 0904, 004 (2009) [arXiv:0901.1750 [hep-ph]]. 
[59] K. P. Modak and D. Majumdar, Astrophys. J. Suppl. 219, no. 2, 37 (2015) [arXiv:1502.05682 [hep-ph]].

[60] K. Jedamzik, Phys. Rev. D 70, 063524 (2004) [astro-ph/0402344].

[61] M. Kawasaki, K. Kohri and T. Moroi, Phys. Rev. D 71, 083502 (2005) [astro$\mathrm{ph} / 0408426]$.

[62] W. Hu and J. Silk, Phys. Rev. Lett. 70, 2661 (1993).

[63] T. Kanzaki, M. Kawasaki, K. Kohri and T. Moroi, Phys. Rev. D 76, 105017 (2007) [arXiv:0705.1200 [hep-ph]].

[64] M. Espirito Santo, K. Hultqvist, P. Johansson, A. Lipniacka, DELPHI 2003-002 PHYS 928 (2003), http://delphiwww.cern.ch/pubxx/delnote/dn2003.html.

[65] G. Abbiendi et al. [OPAL Collaboration], Eur. Phys. J. C 32, 453 (2004) [hepex/0309014].

[66] G. Abbiendi et al. [OPAL Collaboration], Eur. Phys. J. C 35, 1 (2004) [hep-ex/0401026].

[67] A. Pierce and J. Thaler, JHEP 0708, 026 (2007) [hep-ph/0703056 [HEP-PH]].

[68] J. Abdallah et al. [DELPHI Collaboration], Eur. Phys. J. C 31, 421 (2003) [hepex/0311019].

[69] LEP2 SUSY Working Group, $\quad$ LEPSUSYWG/02-04.1, http://lepsusy.web.cern.ch/lepsusy/www/inos_moriond01/charginos_pub.html

[70] J. Alwall et al., JHEP 1407, 079 (2014) [arXiv:1405.0301 [hep-ph]].

[71] K. Cranmer and I. Yavin, JHEP 1104, 038 (2011) [arXiv:1010.2506 [hep-ex]].

[72] M. Acciarri et al. [L3 Collaboration], Phys. Lett. B 482, 31 (2000) [hep-ex/0002043].

[73] P. Abreu et al. [DELPHI Collaboration], "Update of the search for charginos nearly mass-degenerate with the lightest neutralino," Phys. Lett. B 485, 95 (2000) [hepex/0103035].

[74] A. Heister et al. [ALEPH Collaboration], Phys. Lett. B 533, 223 (2002) [hepex/0203020].

[75] G. Abbiendi et al. [OPAL Collaboration], Eur. Phys. J. C 29, 479 (2003) [hepex/0210043].

[76] LEP2 SUSY Working $\quad$ Group, $\quad$ LEPSUSYWG/02-04.1, http://lepsusy.web.cern.ch/lepsusy/www/inoslowdmsummer02/charginolowdm_pub.html

[77] K. Ackerstaff et al. [OPAL Collaboration], "Search for stable and longlived massive charged particles in e+ e- collisions at $\mathrm{s}^{* *}(1 / 2)=130-\mathrm{GeV}-183-\mathrm{GeV}$," Phys. Lett. B 433, 195 (1998) [hep-ex/9803026]. 
[78] P. Abreu et al. [DELPHI Collaboration], "Search for heavy stable and longlived particles in e+ e- collisions at $\mathrm{s}^{* *}(1 / 2)=189-\mathrm{GeV}$," Phys. Lett. B 478, 65 (2000) [hepex/0103038].

[79] G. Abbiendi et al. [OPAL Collaboration], Phys. Lett. B 572, 8 (2003) [hep-ex/0305031].

[80] J. Abdallah et al. [DELPHI Collaboration], Eur. Phys. J. C 38, 395 (2005) [hepex/0406019].

[81] J. Abdallah et al. [DELPHI Collaboration], Eur. Phys. J. C 60, 17 (2009) [arXiv:0901.4486 [hep-ex]].

[82] P. J. Fox, R. Harnik, J. Kopp and Y. Tsai, Phys. Rev. D 84, 014028 (2011) [arXiv:1103.0240 [hep-ph]].

[83] N. Craig, H. K. Lou, M. McCullough and A. Thalapillil, arXiv:1412.0258 [hep-ph].

[84] X. Miao, S. Su and B. Thomas, Phys. Rev. D 82, 035009 (2010) [arXiv:1005.0090 [hep$\mathrm{ph}]$.

[85] M. Gustafsson, S. Rydbeck, L. Lopez-Honorez and E. Lundstrom, Phys. Rev. D 86, 075019 (2012) [arXiv:1206.6316 [hep-ph]].

[86] G. Belanger, B. Dumont, A. Goudelis, B. Herrmann, S. Kraml and D. Sengupta, Phys. Rev. D 91, no. 11, 115011 (2015) [arXiv:1503.07367 [hep-ph]].

[87] G. Aad et al. [ATLAS Collaboration], JHEP 1405, 071 (2014) [arXiv:1403.5294 [hep$\mathrm{ex}]]$.

[88] G. Aad et al. [ATLAS Collaboration], JHEP 1411, 088 (2014) [arXiv:1409.0746 [hep$\mathrm{ex}]]$.

[89] G. Aad et al. [ATLAS Collaboration], Eur. Phys. J. C 75, no. 7, 299 (2015) [Eur. Phys. J. C 75, no. 9, 408 (2015)] [arXiv:1502.01518 [hep-ex]].

[90] T. Sjostrand, S. Mrenna and P. Z. Skands, JHEP 0605, 026 (2006) [hep-ph/0603175].

[91] J. de Favereau et al. [DELPHES 3 Collaboration], JHEP 1402, 057 (2014) [arXiv:1307.6346 [hep-ex]].

[92] A. Denner, S. Dittmaier, T. Kasprzik and A. Mck, Eur. Phys. J. C 73, no. 2, 2297 (2013) doi:10.1140/epjc/s10052-013-2297-x [arXiv:1211.5078 [hep-ph]].

[93] ATLAS Collaboration, ATL-PHYS-PUB-2014-007.

[94] M. Low and L. T. Wang, JHEP 1408, 161 (2014) [arXiv:1404.0682 [hep-ph]].

[95] H. Baer, A. Mustafayev and X. Tata, Phys. Rev. D 89, no. 5, 055007 (2014) [arXiv:1401.1162 [hep-ph]]. 
[96] C. Han, A. Kobakhidze, N. Liu, A. Saavedra, L. Wu and J. M. Yang, JHEP 1402, 049 (2014) [arXiv:1310.4274 [hep-ph]].

[97] G. F. Giudice, T. Han, K. Wang and L. T. Wang, Phys. Rev. D 81, 115011 (2010) [arXiv:1004.4902 [hep-ph]].

[98] S. Gori, S. Jung and L. T. Wang, JHEP 1310, 191 (2013) [arXiv:1307.5952 [hep-ph]].

[99] P. Schwaller and J. Zurita, JHEP 1403, 060 (2014) [arXiv:1312.7350 [hep-ph]].

[100] Z. Han, G. D. Kribs, A. Martin and A. Menon, Phys. Rev. D 89, no. 7, 075007 (2014) [arXiv:1401.1235 [hep-ph]].

[101] H. Baer, A. Mustafayev and X. Tata, Phys. Rev. D 90, no. 11, 115007 (2014) [arXiv:1409.7058 [hep-ph]].

[102] C. Han, D. Kim, S. Munir and M. Park, JHEP 1504, 132 (2015) [arXiv:1502.03734 [hep-ph]].

[103] K. Rolbiecki and K. Sakurai, JHEP 1210, 071 (2012) [arXiv:1206.6767 [hep-ph]].

[104] A. Barr and J. Scoville, JHEP 1504, 147 (2015) [arXiv:1501.02511 [hep-ph]].

[105] Y. Bai and T. M. P. Tait, Phys. Lett. B 710, 335 (2012) [arXiv:1109.4144 [hep-ph]].

[106] N. Weiner and I. Yavin, Phys. Rev. D 86, 075021 (2012) [arXiv:1206.2910 [hep-ph]].

[107] E. Izaguirre, G. Krnjaic and B. Shuve, arXiv:1508.03050 [hep-ph].

[108] B. S. Acharya, K. Boek, C. Pongkitivanichkul and K. Sakurai, JHEP 1502, 181 (2015) [arXiv:1410.1532 [hep-ph]].

[109] S. Gori, S. Jung, L. T. Wang and J. D. Wells, JHEP 1412, 108 (2014) [arXiv:1410.6287 [hep-ph]].

[110] J. Bramante, P. J. Fox, A. Martin, B. Ostdiek, T. Plehn, T. Schell and M. Takeuchi, Phys. Rev. D 91, 054015 (2015) [arXiv:1412.4789 [hep-ph]].

[111] M. Badziak, A. Delgado, M. Olechowski, S. Pokorski and K. Sakurai, arXiv:1506.07177 [hep-ph].

[112] S. D. Thomas and J. D. Wells, Phys. Rev. Lett. 81, 34 (1998) [hep-ph/9804359].

[113] S. Y. Choi, T. Han, J. Kalinowski, K. Rolbiecki and X. Wang, arXiv:1503.08538 [hepph].

[114] M. Berggren, F. Brmmer, J. List, G. Moortgat-Pick, T. Robens, K. Rolbiecki and H. Sert, Eur. Phys. J. C 73, no. 12, 2660 (2013) [arXiv:1307.3566 [hep-ph]].

[115] H. Baer, M. Berggren, J. List, M. M. Nojiri, M. Perelstein, A. Pierce, W. Porod and T. Tanabe, arXiv:1307.5248 [hep-ph]. 
[116] M. Berggren, T. Han, J. List, S. Padhi, S. Su and T. Tanabe, arXiv:1309.7342 [hep-ph].

[117] H. Baer, V. Barger, D. Mickelson, A. Mustafayev and X. Tata, JHEP 1406, 172 (2014) [arXiv:1404.7510 [hep-ph]].

[118] T. Behnke et al., arXiv:1306.6329 [physics.ins-det].

[119] M. Asano et al., Phys. Rev. D 84, 115003 (2011) doi:10.1103/PhysRevD.84.115003 [arXiv:1106.1932 [hep-ph]].

[120] M. Aoki, S. Kanemura and H. Yokoya, Phys. Lett. B 725, 302 (2013) doi:10.1016/j.physletb.2013.07.011 [arXiv:1303.6191 [hep-ph]]. 